\title{
1 Optimal spatial allocation of enzymes as an investment problem
}

\author{
2 Giovanni Giunta, ${ }^{1}$ Filipe Tostevin, ${ }^{1}$ Sorin Tănase-Nicola, ${ }^{2}$ and Ulrich Gerland ${ }^{1, *}$ \\ $3 \quad{ }^{1}$ Physics of Complex Biosystems, Physik-Department, \\ 4 Technische Universität München, James-Franck-Str. 1, 85748 Garching, Germany \\ 5 \\ ${ }^{2}$ Department of Cell and Molecular Biology, Uppsala University, Sweden \\ (Dated: December 15, 2021)
}

\begin{abstract}
Given a limited number of molecular components, cells face various allocation problems demanding decisions on how to distribute their resources. For instance, cells decide which enzymes to produce at what quantity, but also where to position them. Here we focus on the spatial allocation problem of how to distribute enzymes such as to maximize the total reaction flux produced by them in a system with given geometry and boundary conditions. So far, such distributions have been studied by computational optimization, but a deeper theoretical understanding was lacking. We derive an optimal allocation principle, which demands that the available enzymes are distributed such that the marginal flux returns at each occupied position are equal. This 'homogeneous marginal returns criterion' (HMR criterion) corresponds to a portfolio optimization criterion in a scenario where each investment globally feeds back onto all payoffs. The HMR criterion allows us to analytically understand and characterize a localization-delocalization transition in the optimal enzyme distribution that was previously observed numerically. In particular, our analysis reveals the generality of the transition, and produces a practical test for the optimality of enzyme localization by comparing the reaction flux to the influx of substrate. Based on these results, we devise an additive construction algorithm, which builds up optimal enzyme arrangements systematically rather than by trial and error. Taken together, our results reveal a common principle in allocation problems from biology and economics, which can also serve as a design principle for synthetic biomolecular systems.
\end{abstract}

\footnotetext{
* To whom correspondence should be addressed. E-mail: gerland@tum.de
} 


\section{INTRODUCTION}

8 Living systems often need to allocate limited resources to different tasks. For instance, ${ }_{9}$ this situation arises when a colony of microbes differentiates into different cell types, each 10 specialized to a task that contributes to the growth of the colony as a whole [1]. Cells solve 11 this allocation problem by regulating the splitting ratios between cell types during differenti12 ation. Conceptually similar allocation problems arise on the molecular level, where enzymes 13 need to be allocated to different targets. For instance, a limited number of ribosomes must 14 produce different types of proteins in different ratios to achieve balanced cell growth [2-4]. 15 Accordingly, cells dynamically control these ratios when their environment changes to sup16 port different growth rates $[5,6]$. Cells also control the spatial localization of enzymes to 17 optimize or regulate metabolic fluxes [7, 8]. These and many other biological examples share ${ }_{18}$ the basic characteristics of generic resource allocation problems, in which a given amount 19 of a resource must be distributed among competing alternatives to maximize the expected 20 performance of the system [9].

21 The optimal allocation of limited resources has been extensively studied in economics. ${ }_{22}$ Rules such as Kelly's criterion [10] and returns-variance analysis [11] are used to determine 23 optimal betting strategies or optimal portfolios [12]. These criteria typically assume that the ${ }_{24}$ expected return on a bet only depends on its placement and not on the placement of other 25 bets. Although there are biological systems for which such criteria can be directly applied 26 [13], the treatment needs to be generalized to describe systems, for which the performance ${ }_{27}$ depends in coupled, nonlinear ways on the amounts of the resource allocated to each task. ${ }_{28}$ Here we show that the problem of allocating enzymes to different spatial positions to opti29 mize the total enzymatic reaction flux has a one-to-one mapping to an investment problem so where investments and returns are interdependent. In this context, enzyme molecules rep31 resent capital that can be invested at different positions within the system. Each position 32 corresponds to an asset generating a certain expected return in terms of reaction flux. How3з ever, the placement of enzymes affects the substrate profile, and thereby the flux return 34 from other enzymes. Hence, the spatial enzyme allocation problem corresponds to a bet35 ting problem where the coupling between the returns and the bets is determined by the 36 reaction-diffusion dynamics of the substrate.

We consider a general class of models (Fig. 1), in which the substrate $\mathcal{S}$ can enter an 
38 arbitrarily shaped system at (subsections of) the exterior or interior boundaries, while in39 ternal $\mathcal{S}$ is transported via diffusion and possibly also by advection, and $\mathcal{S}$ is lost within 40 the system to competing reactions or at the boundaries due to leakage. The enzymes $\mathcal{E}$ can ${ }_{41}$ be freely arranged on the boundaries and within the system. Potential co-localization of $\mathcal{E}$ 42 with other enzymes $[8,14-16]$ is incorporated in our model to the extent that the substrate 43 influx at the boundaries may be caused by other, boundary-localized enzymes.

44 A previous numerical study of a minimal one-dimensional model within the class of models 45 of Fig. 1 observed a localization-delocalization transition in the optimal enzyme distribution 46 as a function of a dimensionless reaction-diffusion parameter [17]: When reactions are slow ${ }_{47}$ compared to diffusion, localizing all $\mathcal{E}$ at the source boundary is optimal, while a more 48 extended profile with some enzymes also in the interior is optimal for faster reactions. The 49 existence of this transition was subsequently found to be robust with respect to the spatial 50 dimension and specific loss mechanism and reaction kinetics [18]. However, the physical 51 principles governing the optimal configurations and the generality of the transition have 52 remained elusive, and a construction principle for optimal enzyme arrangements is lacking. ${ }_{53}$ Within the economics analogy, the localization-delocalization transition corresponds to a 54 transition in investment strategy from investing everything on a single asset to having a ${ }_{55}$ diversified portfolio with multiple assets. Can we explain the generality of the transition by 56 analogy with a diversification investment strategy? Moreover, just as Kelly's criterion [10] 57 and returns-variance analysis [11] provide a set of rules for the optimal partition of bets, can 58 we derive a generalized criterion to construct optimal enzyme arrangements?

59 We address these open questions with a variational approach, which ultimately reveals 60 the investment strategy underlying optimal enzyme arrangements. The practical significance 61 of our theoretical analysis has three aspects. First, it produces a criterion to determine, for 62 a given experimental situation, whether enzyme localization is optimal. Second, we turn 63 this criterion into an additive construction scheme for optimal enzyme arrangements. This 64 is relevant for synthetic biology [19], where spatial optimization of enzymatic systems is 65 used to boost yields of useful products such as drug molecules [20-22] and biofuels [23, 24]. ${ }_{66}$ Third, the conceptual framework underlying our analysis and the economic interpretation 67 that it provides can be transferred to other biological or economical allocation problems. ${ }_{68}$ This supports the agenda of rationalizing biological strategies with economic principles such 69 as bet-hedging [25-27], division of labor [28], and Pareto fronts [29]. 
A

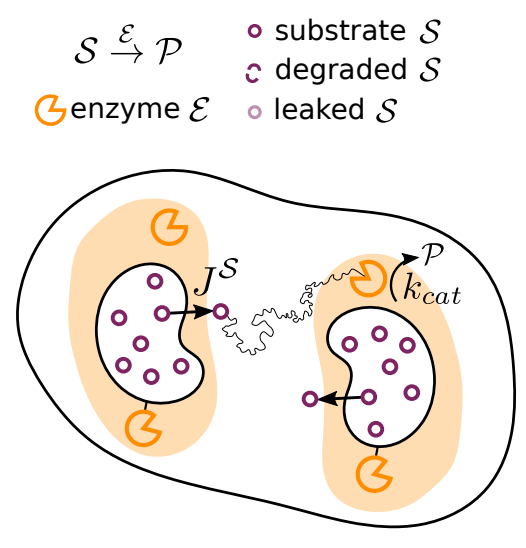

B

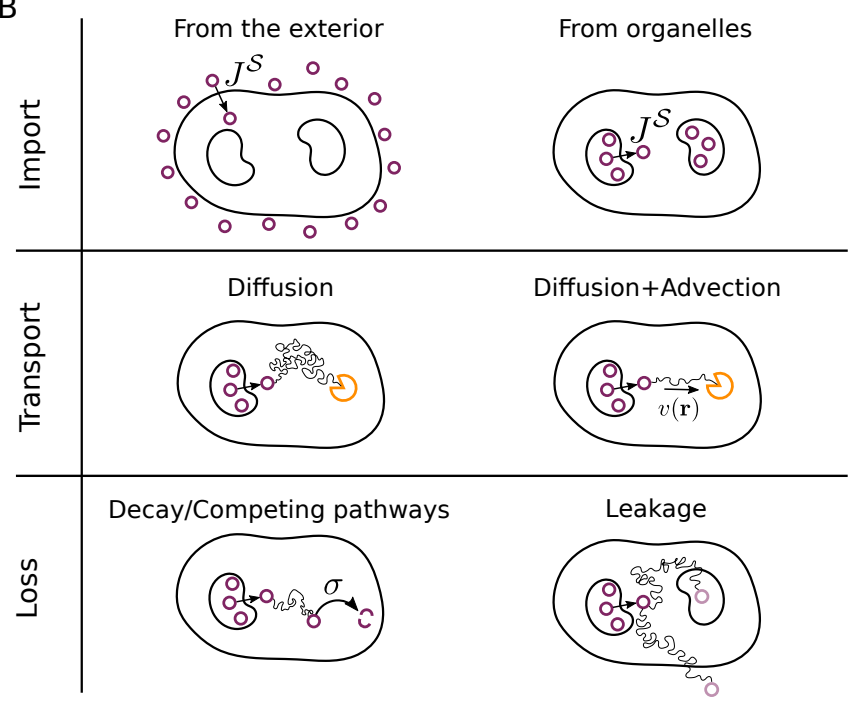

Figure 1. Schematic representation of the class of models considered in this work. (A) An enzyme $\mathcal{E}$ converts a substrate $\mathcal{S}$ to product $\mathcal{P}$ with a catalytic rate $k_{\text {cat }}$. The enzyme molecules can be

freely placed, both within the system and on its interior or exterior membranes. (B-Import) $\mathcal{S}$ is either imported from the exterior or produced within internal compartments. (B-Transport) Within the system, $\mathcal{S}$ is transported diffusively, and possibly also advectively, e.g. by active transport or cytoplasmic streaming. (B-Loss) During transport, $\mathcal{S}$ can also be degraded with rate $\sigma$ or leak out of the system via a membrane.

RESULTS

\section{Variational approach to the spatial enzyme allocation problem}

72 We consider catalytic reactions where a substrate $\mathcal{S}$ is converted into a product $\mathcal{P}$ by 73 an enzyme $\mathcal{E}$ (Fig. 1A). The substrate can enter the system from the exterior or from 74 an internal compartment, e.g. an organelle (Fig. 1B-Import). Enzymes may be located 75 both in the bulk of the sytem and at the boundaries where the substrate enters. The 76 former fraction is described by the concentration field $e(\mathbf{r})$, with $\mathbf{r}$ denoting positions inside 77 the system, while $e_{S}(\mathbf{s})$ is the density of $\mathcal{E}$ on the boundary surface at position s. The ${ }_{78}$ concentration of $\mathcal{S}$ at time $t$ is $\rho(\mathbf{r}, t)$. We consider the distribution of $\mathcal{E}$ as stationary and 79 explore all possible spatial arrangements of the enzymes. The rate at which enzymes locally 
so catalyze product formation depends on the substrate concentration $\rho(\mathbf{r}, t)$. We express this ${ }_{81}$ dependence in the general form $k_{\text {cat }} e F[\rho]$, where $k_{\text {cat }}$ is the catalytic rate of the enzyme and ${ }_{82} F[\rho]$ is a monotonically-increasing reaction function that depends on the enzyme's reaction 83 mechanism, e.g., $F[\rho]=\rho /\left(K_{\mathrm{M}}+\rho\right)$ for Michaelis-Menten kinetics and $F[\rho] \propto \rho$ for linear 84 mass-action kinetics.

85 Within the system, the transport of substrate molecules (Fig. 1B-Transport) can be both 86 stochastic, with a diffusion coefficient $D$, as well as directed, with a velocity field $\mathbf{v}(\mathbf{r})$, 87 where the latter could be due to cytoplasmic streaming [30] or cargo-carrying molecular 88 motors [31]. If the substrate is intrinsically unstable [32] or subject to competing reaction 89 pathways [16], it decays with rate constant $\sigma$ (Fig. 1B-Loss). The dynamical interplay of 9o these processes then follows

$$
\partial_{t} \rho(\mathbf{r}, t)=D \nabla^{2} \rho(\mathbf{r}, t)-\nabla \cdot[\rho(\mathbf{r}, t) \mathbf{v}(\mathbf{r})]-\sigma \rho(\mathbf{r}, t)-k_{\text {cat }} e(\mathbf{r}) F[\rho(\mathbf{r}, t)]
$$

91 In the following, we focus on steady-state conditions, $\partial_{t} \rho(\mathbf{r}, t)=0$, where $\rho(\mathbf{r}, t)=\rho(\mathbf{r})$. We ${ }_{92}$ consider two types of boundaries for the system. First, boundaries at which substrate enters. 93 Including reactions of substrate with enzymes that may be located at such a boundary, we 94 have a boundary condition of the form

$$
j(\mathbf{s})=\left[D \frac{\partial \rho}{\partial \mathbf{n}(\mathbf{s})}-\rho(\mathbf{s}) \mathbf{v}(\mathbf{s}) \cdot \hat{\mathbf{n}}(\mathbf{s})\right]+k_{\mathrm{cat}} e_{S}(\mathbf{s}) F[\rho(\mathbf{s})]
$$

${ }_{95}$ where $j(\mathbf{s})$ is the influx of $\mathcal{S}$ at position $\mathbf{s}, \hat{\mathbf{n}}(\mathbf{s})$ is a unit vector normal to the boundary, 96 pointing outwards from the system, and $\frac{\partial}{\partial \mathbf{n}(\mathbf{s})}$ represents the magnitude of the normal com97 ponent of the gradient. This condition enforces flux conservation at the boundary: the $98 \operatorname{influx} j(\mathbf{s})$ must equal the sum of the local transport and reaction flux. We allow for $j(\mathbf{s})$ to 99 be a discontinuous function of $\mathbf{s}$ as there could be regions on the boundary without influx, ${ }_{100} j(\mathbf{s})=0$ (see Appendix A for details on how such discontinuities are treated). The second 101 kind of boundaries we consider are boundaries at which substrate is lost (Fig. 1B-Loss), e.g. 102 via leakage through a membrane [33]. For these, we impose absorbing boundary conditions, ${ }_{103} \rho(\mathbf{s})=0$. For convenience, we represent these boundary conditions as

$$
0=k(\mathbf{s})\left\{j(\mathbf{s})-\left[D \frac{\partial \rho}{\partial \mathbf{n}(\mathbf{s})}-\rho(\mathbf{s}) \mathbf{v}(\mathbf{s}) \cdot \hat{\mathbf{n}}(\mathbf{s})\right]-k_{\mathrm{cat}} e_{S}(\mathbf{s}) F[\rho(\mathbf{s})]\right\}-h(\mathbf{s}) \rho(\mathbf{s})
$$

104 where $k(\mathbf{s})$ and $h(\mathbf{s})$ are set to represent absorbing boundaries $(k=0, h=1)$ or boundaries 105 with influx of substrate $(k=1, h=0)$. 
The interplay of processes of the type illustrated in Fig. 1 and described by Eqs. 1 to 3 107 have been demonstrated to generate stable gradients of intracellular components in a variety 108 of biological contexts [34-37]. An immediate consequence of non-uniform substrate profiles 109 is that different spatial enzyme distributions $e(\mathbf{r})$ generate different total reaction fluxes. In 110 cases where the substrate is produced inside organelles, e.g. mitochondria or endoplasmatic 111 reticula, the associated enzymes tend to be concentrated in proximity of the organelle mem112 brane but also present in the cytoplasm [38-41]. In contrast, when the substrate is imported 113 into the cytoplasm from the external environment, some of the associated metabolic enzymes 114 are localized to the external membrane [42].

${ }_{115}$ The total reaction flux $J^{\mathcal{P}}$, i.e. the rate at which the whole system produces $\mathcal{P}$, has 116 contributions from enzymes at the boundaries and within the bulk,

$$
J^{\mathcal{P}}=\int_{S} k_{\text {cat }} e_{S}(\mathbf{s}) F[\rho(\mathbf{s})] \mathrm{d} \mathbf{s}+\int_{V} k_{\text {cat }} e(\mathbf{r}) F[\rho(\mathbf{r})] \mathrm{d} \mathbf{r},
$$

117 where the first integral is over all boundary surfaces $S$ and the second is over the volume $V$ 118 of the system. Given a fixed amount of available enzymes, $E_{T}=\int_{S} e_{S}(\mathbf{s}) \mathrm{d} \mathbf{s}+\int_{V} e(\mathbf{r}) \mathrm{d} \mathbf{r}$, how 119 should these enzymes be positioned such as to maximize the rate $J^{\mathcal{P}}$ of product formation? 120 This optimization problem can be approached by defining a functional of the form $\mathcal{L}=$ ${ }_{121} J^{\mathcal{P}}-\sum_{i} \lambda_{i} C_{i}$, where $C_{i}$ represent different constraints with associated Lagrange multipliers ${ }_{122} \lambda_{i}$. For our model, these constraints are that (i) the total amount of enzymes must equal ${ }_{123} E_{T}$, (ii) $\rho(\mathbf{r})$ and $e(\mathbf{r})$ must jointly satisfy the reaction-diffusion-advection equation, Eq. 1, 124 at each point $\mathbf{r}$, and (iii) $\rho(\mathbf{s})$ and $e_{S}(\mathbf{s})$ must jointly satisfy the boundary condition, Eq. 3, 125 at each boundary point $\mathbf{s}$. The resulting Lagrangian has the form

$$
\begin{aligned}
\mathcal{L}= & J^{\mathcal{P}}-\lambda_{e}\left[\int_{S} e_{S}(\mathbf{s}) \mathrm{d} \mathbf{s}+\int_{V} e(\mathbf{r}) \mathrm{d} \mathbf{r}-E_{T}\right] \\
& +\int_{V} \lambda_{V}(\mathbf{r}) \mathcal{D}[e(\mathbf{r}), \rho(\mathbf{r}), \mathbf{r}] \mathrm{d} \mathbf{r}+\int_{S} \lambda_{S}(\mathbf{s}) \mathcal{B}\left[\rho(\mathbf{s}), e_{S}(\mathbf{s}), \mathbf{s}\right] \mathrm{d} \mathbf{s},
\end{aligned}
$$

126 where $\mathcal{D}[e(\mathbf{r}), \rho(\mathbf{r}), \mathbf{r}]$ and $\mathcal{B}\left[\rho(\mathbf{s}), e_{S}(\mathbf{s}), \mathbf{s}\right]$ represent the right-hand side of Eqs. 1 and 3 127 respectively, and the signs in front of the Lagrange multipliers have been chosen such that ${ }_{128}$ they result to be positive. Maximizing $\mathcal{L}$ with respect to $e_{S}(\mathbf{s})$ and $e(\mathbf{r})$ while simultaneously 129 minimizing with respect to all Lagrange multipliers yields the optimal enzyme arrangements 130 satisfying the constraints.

${ }_{131}$ The optimal enzyme arrangement may consist of both regions with a finite density of 132 enzymes and regions with no enzymes. Indeed, previous numerical studies have shown that 
133 the optimal enzyme distributions are often discontinuous [16-18]. To account for such dis134 continuities, we split the volume $V$ into different sub-spaces (and similarly for the boundaries ${ }_{135} S$ ); within each sub-space the enzyme density must be continuous and $e(\mathbf{r}) \geq 0$, but at the 136 interfaces continuity is not required. The number of sub-spaces as well as the shape of the 137 interface between each pair of sub-spaces are then also variables to be optimized (see Ap${ }_{138}$ pendix A for details and Appendix B, E, F for examples). This is equivalent to applying the 139 Karush-Kuhn-Tucker conditions $[43,44]$ on the Lagrangian, Eq. 5, while imposing $e(\mathbf{r}) \geq 0$. ${ }_{140}$ We will see below that the variational approach is useful even when it is impossible to 141 solve for the optimal enzyme arrangement analytically. In cases where it is possible to ${ }_{142}$ extract the exact functional form of the optimal profile, the problem can usually be reduced 143 to a low-dimensional optimization over a small number of parameters, which is then easily 144 performed numerically (see Appendix E for an example). For systems where the functional 145 form of $e(\mathbf{r})$ cannot be found analytically, such as those with complex geometries, we will ${ }_{146}$ describe a scheme for constructing the optimal enzyme arrangement.

\section{Enzyme allocation problem as a betting game}

${ }_{148}$ The spatial enzyme allocation problem defined above can be mapped to a betting game. ${ }_{149}$ We consider a game that has a single winning outcome out of a set of mutually exclusive 150 events. Each outcome $i$ has a certain probability $p_{i}$ of being the winning event. We assume 151 that a gambler bets a total amount $B=\sum_{i} b_{i}$ with $b_{i} \geq 0$ denoting the bet on outcome $i$. 152 If $i$ is the winning event, the gambler wins the amount $\alpha_{i} b_{i}$, with $\alpha_{i} \geq 0$ referred to as the 153 odds of event $i$. The expected amount of capital won by the gambler is thus $C=\sum_{i} \alpha_{i} b_{i} p_{i}$. ${ }_{154}$ If the expected odds $\alpha_{i} p_{i}$ are independent of the bets $b_{i}$, the optimal strategy for a given 155 total budget $B$ is to invest everything in the outcome with highest expected odds. However, 156 if the expected odds are coupled to the bets, the optimization of $C$ subject to the budget 157 constraint becomes nontrivial, and diversification of bets may be the optimal strategy.

${ }_{158}$ By comparing the objective function $C$ of the betting game to the reaction flux $J^{\mathcal{P}}$ of 159 our spatial enzyme allocation problem, we see that the set of events $\{i\}$ maps to the set of 160 possible enzyme positions, a bet $b_{i}$ corresponds to the enzyme concentration $e(\mathbf{r})$ allocated ${ }_{161}$ to $\mathbf{r}$, and the fixed budget $B$ corresponds to the total enzyme amount $E_{T}$. Moreover, the 162 probability of $i$ being the winning event maps to the probability density for a substrate 
${ }_{163}$ molecule to bind to an enzyme at position $\mathbf{r}, p_{i} \leftrightarrow F[\rho(\mathbf{r})]$, and the odds $\alpha_{i}$ correspond to 164 the catalytic rate $k_{\text {cat }}$.

165 In the enzyme system, part of the substrate is lost via the mechanisms of Fig. 1B-Loss, 166 which can be included in the betting game via events with finite probability but zero odds. ${ }_{167}$ However, the objective function $C=\sum_{i} \alpha_{i} b_{i} p_{i}$ needs to be generalized to account for the 168 nonlinearity and spatial coupling in the enzyme system: The "expected odds" $k_{\text {cat }} F[\rho(\mathbf{r})]$ 169 depend on the "bets" $e(\mathbf{r})$, since the binding between enzymes and substrate at a given 170 location generates a diminishing returns effect, i.e., the addition of extra enzymes at that 171 position becomes gradually less efficient. Moreover, the substrate concentrations at different 172 positions are coupled, due to the substrate diffusion (and possibly advection), such that the 173 substrate concentration at one position ultimately depends on the enzymes at every position. ${ }_{174}$ This means that the bets placed on one event $i$ also affect the winning probabilities $p_{j}$ with ${ }_{175} j \neq i$. These effects are captured by making the probabilities $p_{i}$ a function of the vector of 176 bets $p_{i}=f_{i}(\mathbf{b})$ and adding the feedback from the bets onto the winning probabilities via 177 constraints in the objective function,

$$
\mathcal{C}=\sum_{i} \alpha_{i} b_{i} p_{i}-\sum_{i} \lambda_{i}\left[f_{i}(\mathbf{b})-p_{i}\right]-\lambda\left(\sum_{i} b_{i}-B\right),
$$

178 where $\lambda_{i}$ are the Lagrange multipliers for the feedback constraint, analogous to $\lambda(\mathbf{r})$ in the 179 Lagrangian $\mathcal{L}$ of the enzyme system, Eq. 5. The behavior of this generalized betting game 180 depends on the form of $f_{i}(\mathbf{b})$. In Appendix H, we show for example that in a simple game 181 with two possible winning outcomes, a diminishing returns effect $\left(\partial f_{i} / \partial b_{i}<0\right)$ can result in 182 an optimal strategy with diversified bets $\left(b_{1}, b_{2}>0\right)$.

183 The above mapping illustrates that in order to solve our optimal enzyme allocation prob$184 \mathrm{lem}$, we need to generalize the treatment of betting games to include the coupling between 185 bets and expected gains. Note that we considered only a single round of the betting game so 186 far. Classical treatments of betting games, e.g. the one by Kelly [10], often assume that the 187 gambler iteratively reinvests a certain fraction of the current capital, such that the long-term 188 capital growth rate must be optimized rather than $C$. In the enzyme context, this would 189 correspond to a situation where the product of the enzymatic reaction is used to generate 190 additional enzymes to be placed in the system. Here, we first treat the stationary enzyme 191 allocation problem, corresponding to a repetitive game in which the gambler bets the same 192 total amount $B$ in every round, such that the optimal strategy is the same as the optimal 
193 strategy for a single round. We will later see that our scheme to construct optimal strate194 gies can also be adapted to the case where a constant fraction of the capital is iteratively 195 reinvested (see below and Appendix H).

\section{Optimal allocation principle}

197 We now leverage the above variational approach to solve the enzyme allocation problem. 198 Towards this end, we examine the variation of the Lagrangian, Eq. 5, with respect to the 199 enzyme density,

$$
\frac{\delta \mathcal{L}}{\delta e(\mathbf{r})}=\underbrace{k_{\mathrm{cat}} F[\rho(\mathbf{r})]-\lambda_{V}(\mathbf{r}) k_{\mathrm{cat}} F[\rho(\mathbf{r})]}_{\equiv \frac{d J^{\mathcal{P}}}{d e(\mathbf{r})} \text { "marginal return" }}-\lambda_{e} .
$$

200 This variation corresponds to the change in the constrained flux $\mathcal{L}$ as enzymes are added at 201 position $\mathbf{r}$. Three different terms contribute to this change. The first is the increase in flux 202 that would be observed upon adding enzymes at position $\mathbf{r}$ if everything else in the system 203 were to remain unaffected, which corresponds mathematically to $\frac{\delta J^{\mathcal{P}}}{\delta e(\mathbf{r})}$. However, changing $204 e(\mathbf{r})$ also alters the substrate density profile $\rho(\mathbf{r})$, thereby affecting the rate of reactions at 205 all positions. This coupling is captured by the second term in Eq. 7, where the Lagrange 206 multiplier $\lambda_{V}(\mathbf{r})$ ensures that $\rho(\mathbf{r})$ and $e(\mathbf{r})$ satisfy the constraint of the reaction-diffusion 207 equation. Thus the sum of the first two terms is the total rate of change of the reaction 208 flux as extra enzymes are added at position $\mathbf{r}$. In the following, we refer to this quantity 209 as the "marginal return" on an investment of enzymes, and denote it by $\frac{d J^{\mathcal{P}}}{d e(\mathbf{r})}$. Finally, the 210 third term, $-\lambda_{e}=-\frac{\partial \mathcal{L}}{\partial E_{T}}$, corresponds to a marginal cost of adding extra enzymes into the 211 system. This marginal cost can be interpreted as the reduction in flux from other positions ${ }_{212} \mathbf{r}^{\prime} \neq \mathbf{r}$ in the optimal configuration, as enzymes are moved from these positions to $\mathbf{r}$ in order 213 to satisfy the constraint of constant total enzyme number.

214 In the optimal enzyme profile $e^{*}(\mathbf{r})$, we must distinguish between regions where placing 215 enzymes is optimal $\left(e^{*}(\mathbf{r})\right.$ finite $)$, and empty regions where placing enzymes is suboptimal ${ }_{216}\left(e^{*}(\mathbf{r})=0\right)$. Wherever $e^{*}(\mathbf{r})>0$, the variation $\left.\frac{\delta \mathcal{L}}{\delta e(\mathbf{r})}\right|_{e^{*}}=0$. The functional derivative with 217 respect to the surface density $\frac{\delta \mathcal{L}}{\delta e_{S}(\mathbf{s})}$ has the same form as Eq. 7. Thus the optimal profile ${ }_{218}$ follows a 'homogeneous marginal returns' criterion (HMR criterion): it is such that at any 219 position with enzymes, the marginal return on placed enzymes equals a constant value, 220 namely $\left.\frac{d J^{\mathcal{P}}}{d e(\mathbf{r})}\right|_{e^{*}}=\lambda_{e}$. By contrast, in empty regions, the marginal flux gain from placing 
221 enzymes is less than the associated marginal cost, $\left.\frac{d J^{\mathcal{P}}}{d e(\mathbf{r})}\right|_{e^{*}}<\lambda_{e}$.

${ }_{222}$ To illustrate this optimal allocation principle, we apply the HMR criterion to the simple 223 case of a linear reaction $\left(F[\rho]=\rho / K_{\mathrm{M}}\right)$ with no advection or decay $(|\mathbf{v}|=\sigma=0)$ in a 224 one-dimensional domain of size $L$, with a source of substrate at the left boundary $(x=0)$ 225 and an absorbing boundary on the right (Fig. 2A). This case is exactly solvable within the 226 variational approach, such that we can explicitly see how variations of the enzyme profile 227 affect the marginal returns landscape. Moreover, this case allows us to cross-validate our 228 variational approach with a previous numerical study [17]. As shown in Appendix B, the 229 optimal enzyme profile for this example is

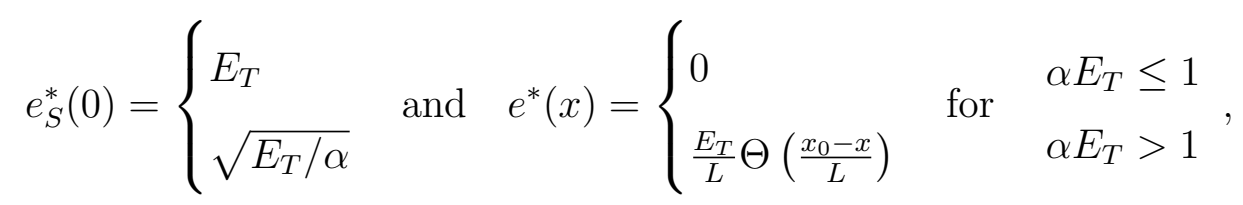

230 with $\alpha=L k_{\text {cat }} /\left(K_{\mathrm{M}} D\right)$, the Heaviside step function $\Theta(x)$, and $x_{0}=L\left[1-\left(\alpha E_{T}\right)^{-1 / 2}\right]$. ${ }_{231}$ Eq. 8 shows that the optimal enzyme profile undergoes a localization-delocalization transi232 tion from having all enzymes localized at the source, to an extended profile with enzymes 233 also in the interior of the system (Fig. 2B, orange lines). The transition occurs as a function 234 of the dimensionless parameter $\alpha E_{T}$, with $\alpha E_{T}=1$ marking the transition point.

235 The analytic solution demonstrates how the optimal enzyme profile arises from a balance 236 between gain and cost. The shape of the marginal returns landscape (Fig. 2B, green lines) 237 changes as the total enzyme amount $E_{T}$ is increased. Below the transition, the marginal 238 returns $\frac{d J^{\mathcal{P}}}{d e(x)}$ strictly decrease with distance from the source, i.e., the maximal value occurs 239 only at $x=0$, whereas above the transition the marginal returns take on a constant value 240 over the region $0 \leq x \leq x_{0}$ where enzymes are present and decreases only for $x>x_{0}$. The 241 level of this marginal returns plateau corresponds to the marginal cost $\lambda_{e}$ of adding extra 242 enzymes into the system (Fig. 2C, middle). This marginal cost decreases with increasing 243 availability of enzymes (increasing $E_{T}$ ). Fig. $2 \mathrm{C}$ shows how the shape of the marginal returns 244 landscape changes when the enzyme profile deviates from the optimum. If it deviates by 245 having a larger proportion of enzymes located at the source (Fig. 2C, top), the marginal 246 returns landscape becomes non-monotonic with a peak at a position $x>0$. The elevated 247 enzyme density in this region depletes the substrate locally, reducing the turnover by each 248 enzyme and diminishing the returns from adding additional enzymes. The peak in the 249 marginal returns landscape implies that a higher flux can be achieved by moving some 
A

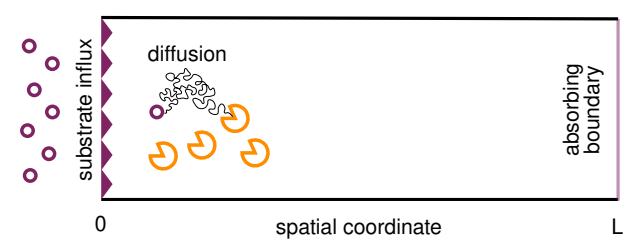

B

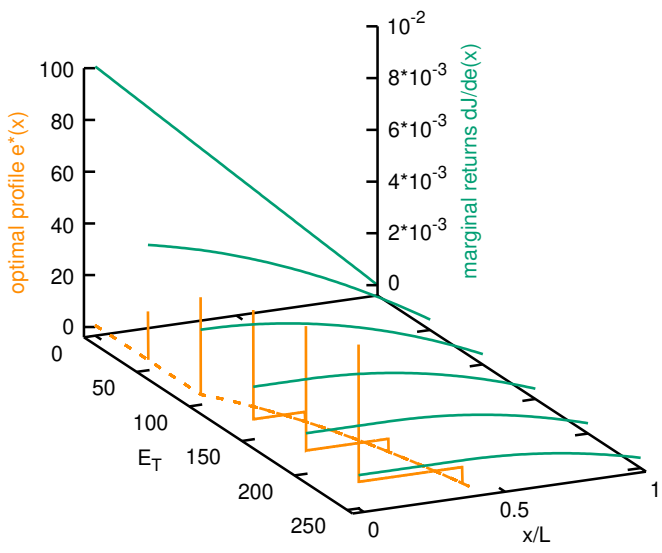

C
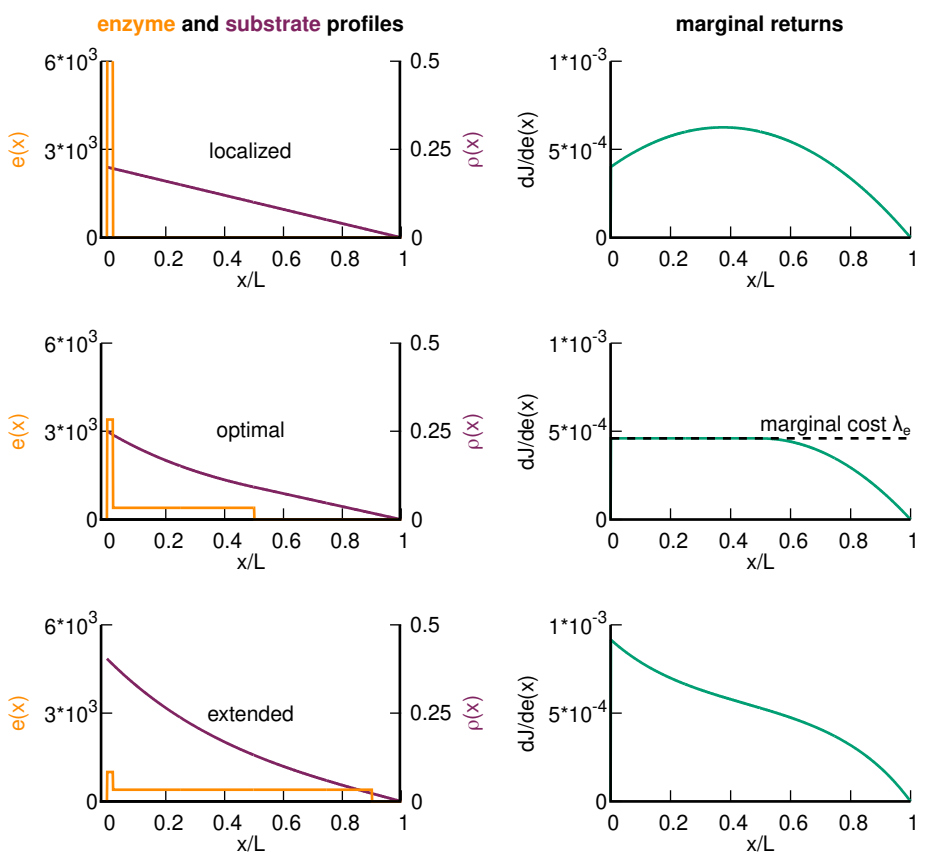

Figure 2. Homogeneous Marginal Returns (HMR) criterion for the optimal spatial allocation of enzymes. (A) Illustration of the analytically solvable 1D model with a source of substrate at $x=0$ and an absorbing boundary at $x=L$. Within the system, the substrate diffuses and can react with the spatially arranged enzymes. (B) Optimal enzyme profile with corresponding marginal return landscape as a function of the total enzyme amounts $E_{T}$ (the reaction-diffusion parameter is fixed to $\alpha=10^{-2}$ ). (C) Different enzyme and substrate profiles (left) with corresponding marginal returns (right) for $E_{T}=400$. Top: Localized configuration. The marginal returns landscape is peaked at $x>0$, indicating that enzymes should be moved away from the source. Middle: Optimal profile with constant marginal return over the region with enzymes (HMR criterion). In the constant region, the marginal return is equal to the marginal cost $\lambda_{e}$ of adding extra enzymes into the system. Bottom: Overextended profile. Monotonically decreasing marginal return, indicating that enzymes should be moved towards the source at $x=0$.

250 enzymes away from the source. In contrast, if the enzyme profile is overextended (Fig. 2C, 251 bottom), the marginal returns landscape has a maximum at the source, such that the flux 252 can be increased by moving enzymes towards the source.

253 Note that for the 1D problem analyzed in Fig. 2, the single source at $x=0$ is always the 254 position with the highest substrate concentration, independently of the enzyme arrangement 255 (see purple lines). Hence an individual enzyme would always optimize its own productivity 
256 at $x=0$. However, for $\alpha E_{T}>1$, the total productivity of the population of enzymes is 257 optimized only when some enzymes are located at positions $x>0$ with non-maximal sub258 strate concentrations. The optimal profile is thus the result of a bet-hedging strategy: Some 259 productivity of individual enzymes is sacrificed to optimize the reaction flux produced by the 260 entire population of enzymes. How relevant is the transition for biological systems? Given 261 that the ratio $k_{\text {cat }} / K_{\mathrm{M}}$ varies by orders of magnitude, $10^{2}-10^{9}(\mathrm{Ms})^{-1}$ [45], the corresponding ${ }_{262} \alpha=L k_{\text {cat }} /\left(K_{\mathrm{M}} D\right)$ for a system with $L=1 \mu \mathrm{m}, D=100 \mu \mathrm{m}^{2} / \mathrm{s}$ is in the range from $10^{-7}$ 263 to 1 , such that the transition can occur for enzyme numbers $E_{T}^{t}$ in the range 1-10 ${ }^{7}$. For ${ }_{264}$ comparison, the range of protein copy numbers in a yeast cell is $1-10^{6}$ [46], suggesting that 265 different enzymatic systems cover both sides of the transition.

\section{General condition for transitions in the optimal enzyme arrangement}

267 Transitions in the optimal enzyme arrangement of the above type, from a regime in which ${ }_{268}$ enzymes are colocalized with the source of $\mathcal{S}$ to a regime where enzymes are distributed 269 within the system, have been observed in a range of reaction-diffusion models [18]. We 270 therefore asked whether there was an underlying principle determining when such transitions 271 occur, and whether it can be generalized, e.g. to systems with more complex geometries.

272 Fig. 2 suggests that the marginal returns landscape leads to a general condition for 273 localization-delocalization transitions. For $E_{T}$ below the transition value, the landscape 274 is strictly decreasing, with the position generating the highest returns coinciding with the 275 source of substrate. As the transition is reached and passed, the landscape becomes flat as 276 positions in the vicinity of the source begin to generate the same returns as the source posi277 tion. This behavior generalizes to higher dimensional systems (see Appendix D): Enzymes 278 should be placed only at the source on the surface and not in the interior, as long as

$$
\frac{\partial}{\partial \mathbf{n}(\mathbf{s})} \frac{d J^{\mathcal{P}}}{d e}>0
$$

279 corresponding to a negative slope of the marginal returns landscape from the source at $\mathbf{s}$ 280 into the interior of the system, as in Fig. 2B for $E_{T}<\alpha^{-1}$. By contrast, if Eq. 9 evaluated 281 at $\mathbf{S}$ becomes an equality, the positions adjacent to $\mathbf{S}$ generate the same returns as $\mathbf{S}$, so that 282 the optimal enzyme profile features enzymes both at the boundary $\mathbf{s}$ and in the interior of 283 the system adjacent to $\mathbf{s}$. 


\begin{tabular}{|c|c|c|c|c|c|}
\hline $\begin{array}{l}\text { Reaction } \\
\text { flux jP/j }\end{array}$ & $\begin{array}{c}\text { Diffusive } \\
\text { flux j } j^{D} / j\end{array}$ & $\begin{array}{l}\text { Geometry/ } \\
\text { Symmetry }\end{array}$ & Reaction & $\sigma$ & v \\
\hline $\begin{array}{l}\bar{v}_{\bar{v}} \\
\end{array}$ & 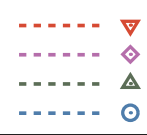 & $\begin{array}{l}\text { 3D spherical } \\
\text { 1D } \\
(1 \mathrm{D} \text { two sources } \\
\text { 1D two sources }\end{array}$ & $\begin{array}{l}\text { M.-M. } \\
\text { Linear } \\
\text { Linear } \\
\text { Linear }\end{array}$ & $\begin{array}{l}\mathrm{N} \\
\mathrm{N} \\
\mathrm{Y} \\
\mathrm{Y}\end{array}$ & $\begin{array}{l}\mathrm{N} \\
\mathrm{Y} \\
\mathrm{N} \\
\mathrm{N}\end{array}$ \\
\hline
\end{tabular}

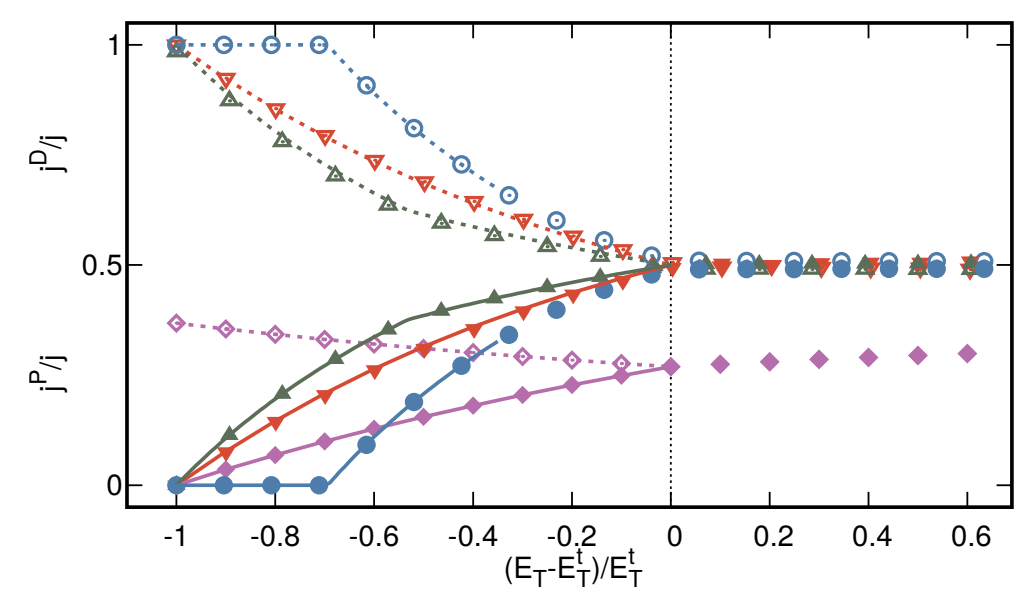

Figure 3. The local reaction flux, $j^{\mathcal{P}}(\mathbf{s})$, and net diffusive flux, $j^{D}(\mathbf{s})$, as a fraction of $\mathcal{S}$ influx, $j(\mathbf{s})$, are plotted for different models against the amount of enzymes in the system, $E_{T}$, rescaled by the transition amount, $E_{T}^{t}$. In all cases, $j^{D}>j^{\mathcal{P}}$ until they coincide at the transition point, as predicted by Eq. 10 . When $\mathbf{v}=0, j^{\mathcal{P}}=j^{D}=j / 2$ at and above the transition. Lines show analytical solutions of the constrained optimization. Points show results of numerical optimizations computed either by solving numerically an analytically-derived optimization condition (Appendix E), or using the construction algorithm. For the two-source model, the two curves show the two unequal sources, each rescaled with the corresponding $E_{T}^{t}$ value; see Appendix F and Fig. F.1 for a detailed analysis of this model.

${ }_{284}$ The abstract mathematical condition Eq. 9 can be turned into an experimentally mean285 ingful condition (see Appendix D) by expressing it as a comparison between the local net ${ }_{286}$ diffusive flux $j^{D}(\mathbf{s})$ of $\mathcal{S}$ away from the surface, and the local flux $j^{\mathcal{P}}(\mathbf{s})$ of product formation 287 due to surface enzymes,

$$
j^{D}(\mathbf{s}) \equiv D \frac{\partial \rho}{\partial \mathbf{n}(\mathbf{s})}>k_{\mathrm{cat}} e_{S}(\mathbf{s}) F[\rho(\mathbf{s})] \equiv j^{\mathcal{P}}(\mathbf{s}) .
$$

288 If this inequality holds, no enzymes should be placed in the interior of the system in the 289 vicinity of $\mathbf{s}$. For low amounts of enzymes, the optimal strategy is to place enzymes at 290 position s to counter the diffusive flux $j^{D}(\mathbf{s})$. This is optimal up to the point at which the 291 diffusive flux $j^{D}(\mathbf{s})$ equals the reaction flux $j^{\mathcal{P}}(\mathbf{s})$, which defines the transition point of the 
292 localization-delocalization transition.

293 Further combining Eq. 10 with the boundary condition Eq. 2 yields $j^{\mathcal{P}}(\mathbf{s})<(j(\mathbf{s})-$ $\left.{ }_{294} \rho(\mathbf{s}) \mathbf{v}(\mathbf{s}) \cdot \hat{n}(\mathbf{s})\right) / 2$, which simplifies in the case of no advection $(\mathbf{v}=0)$ to $j^{\mathcal{P}}<j(\mathbf{s}) / 2$. Thus, 295 in systems with purely diffusive $\mathcal{S}$ transport, a localization-delocalization transition occurs 296 when the reaction flux equals exactly half the influx of $\mathcal{S}$. Note that Eq. 10 is general, 297 independent of $\mathbf{v}$ and $\sigma$ and other details of the system in question. It is required only 298 that the boundary with influx follows Eq. 2. Other aspects of the model will affect $\rho(\mathbf{r})$, 299 but not the form of Eq. 10, showing that the diffusive motion of $\mathcal{S}$ and the strength of the 300 reaction are the crucial determinants of the transition point. To illustrate this generality, 301 we compared the fluxes $j^{D}(\mathbf{s})$ and $j^{\mathcal{P}}(\mathbf{s})$ as $E_{T}$ was varied, in systems with different spatial 302 dimensions as well as systems with and without decay and advection of $\mathcal{S}$. As shown in ${ }_{303}$ Fig. 3, in all cases both Eq. 10 below the transition value of $E_{T}$ and equality above the 304 transition were satisfied.

305 The inequality condition Eq. 10 is a strictly local condition. In systems with multiple or 306 spatially varying sources of substrate there will in general not be a single global transition 307 but rather a sequence of local transitions at different positions $\mathbf{s}$ at different $E_{T}$ levels. We 308 therefore investigated a system with two unequal sources of $\mathcal{S}$. In such a system the optimal 309 enzyme profile typically undergoes three transitions (see Fig. F.1). For small $E_{T}$, enzymes 310 accumulate where the influx of $\mathcal{S}$ is the highest. As $E_{T}$ is increased, the marginal returns 311 for placing enzymes in the vicinity of the two sources of $\mathcal{S}$ become more similar, until at a 312 threshold value of $E_{T}$ they become equal. Above this value of $E_{T}$, enzymes accumulate at 313 both sources in a configuration with two unequal clusters. Increasing $E_{T}$ further, a second 314 transition is reached where the optimal enzyme profile begins to extend from the stronger 315 source into the system. Finally, at yet larger $E_{T}$, the optimal enzyme profile begins to 316 extend also from the weaker source. For the latter two transitions, at which the optimal 317 enzyme arrangement changes from membrane-localized to extended, we confirmed that the 318 inequality Eq. 10 held below the transition point and equality above (Fig. 3 green and blue).

\section{Geometrical interpretation and analogy to portfolio selection}

320 Our optimal enzyme allocation problem has a geometrical interpretation in the space 321 of (discretized) enzyme configurations e. A component $e_{i}$ of the vector e corresponds to 


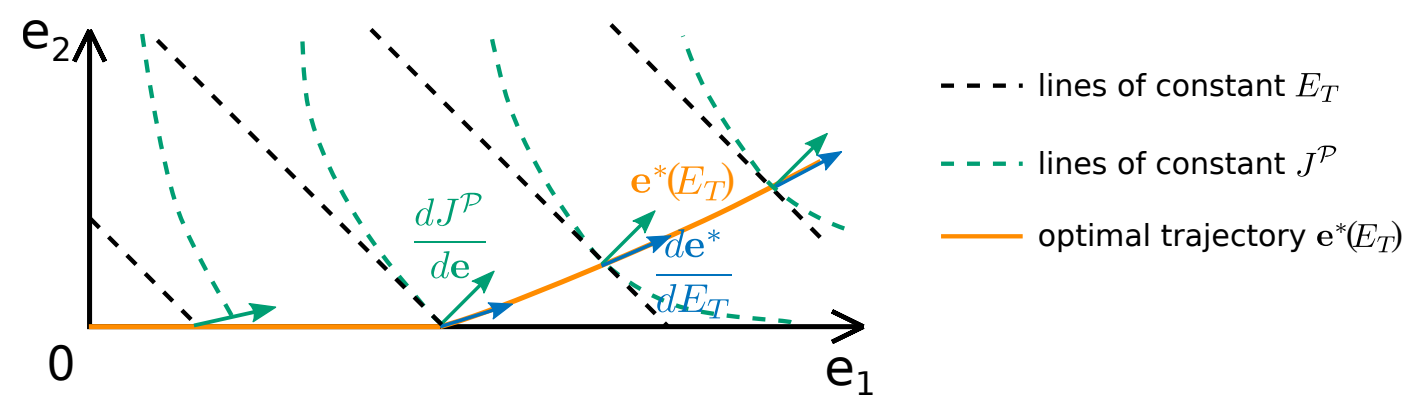

Figure 4. Geometrical analysis of the optimal enzyme configuration in a system with two sites. Dashed black lines show lines of constant $E_{T}=e_{1}+e_{2}$, while dashed green lines show lines of constant reaction flux $J^{\mathcal{P}}$. The optimal configuration $\mathbf{e}^{*}=\left(e_{1}^{*}, e_{2}^{*}\right)$ for each value of $E_{T}$ forms an optimal trajectory $\mathbf{e}^{*}\left(E_{T}\right)$ (orange). Green arrows show the marginal returns vector $\frac{d J^{\mathcal{P}}}{d \mathbf{e}}$. The tangent vector $\frac{d \mathbf{e}^{*}}{d E_{T}}$ (blue arrows) shows how added enzymes should be optimally partitioned between the two sites.

322 the amount of enzymes allocated to position $i$, and the dimension of e to the number of 323 possible enzyme positions. Hence, the subspace with constant total enzyme amount $E_{T}$ is 324 the hyperplane intersecting each axis at $e_{i}=E_{T}$. Each point in the enzyme configuration 325 space has an associated flux $J^{\mathcal{P}}$, and the allocation problem amounts to finding the point of 326 maximal flux within the hyperplane.

$327 \quad$ Fig. 4 illustrates the geometrical problem for the simplest case with only two possible 328 enzyme positions (here, we assume a substrate source at position one, and loss of substrate 329 via one of the loss mechanisms of Fig. 1B). In Fig. 4, $E_{T}$ hyperplanes are indicated by ззо dashed black lines, while the dashed green curves are lines of constant flux $J^{\mathcal{P}}$. Since 331 the marginal returns vector $\frac{d J^{\mathcal{P}}}{d \mathbf{e}}$ is the flux gradient, the lines of constant flux must be 332 locally perpendicular to it. Adding extra enzymes at any location with nonzero substrate 333 concentration will increase the total reaction flux, implying that the direction of the marginal 334 returns vector always lies in the first quadrant (all components positive). Furthermore, the 335 direction must turn clockwise as we move along a line of constant flux in the direction of 336 increasing $e_{2}$, since the second component of the marginal returns vector must decrease as 337 we allocate a larger fraction of enzymes to position two, while the first component must 338 increase. Hence, the lines of constant flux are convex.

339 Geometrically, the solution of the optimal allocation problem corresponds to the touching 340 point $\mathbf{e}^{*}$ between a given $E_{T}$ line and the $J^{\mathcal{P}}$ line with the largest $J^{\mathcal{P}}$ value that still touches 
${ }_{341}$ the $E_{T}$ line. At small $E_{T}$ values, the $J^{\mathcal{P}}$ line is steeper than the $E_{T}$ line, such that $\mathbf{e}^{*}\left(E_{T}\right)$ 342 lies on the $e_{1}$ axis (orange line in Fig. 4). However, at a certain threshold value of $E_{T}$, the ${ }_{343} E_{T}$ line becomes tangential to the $J^{\mathcal{P}}$ line. At this point, the marginal returns from position 3441 and 2 become equal, i.e., the marginal returns vector points in the $(1,1)$ direction. When ${ }_{345} E_{T}$ is further increased, the optimal path $\mathbf{e}^{*}\left(E_{T}\right)$ departs from the $e_{1}$ axis and follows the 346 tangent point between the $E_{T}$ and $J^{\mathcal{P}}$ lines, maintaining equal marginal returns. Note that, 347 due to the convexity of the lines of constant $J^{\mathcal{P}}$, this tangent point is the global optimum 348 at a given $E_{T}$ value.

349 The above geometrical interpretation is somewhat analogous to Markowitz' geometrical 350 analysis of investment portfolio optimization [11]. Markowitz considered the problem of 351 minimizing the variance of a portfolio of investments at a constant level of returns (returns352 variance analysis). The space of portfolios, which is determined by the relative amounts in353 vested in different securities, is analogous to the space of enzyme configurations e. Markovitz 354 noted that optimal portfolios are tangent points between lines of constant expected returns 355 and lines of constant variance. One key difference to our case is that the returns are indepen356 dent of the portfolio choice in Markovitz' case, leading to simple straight lines of constant 357 returns in the space of portfolios. Another key difference is that the variance of the returns 358 has played no role in our analysis so far, since we considered enzymes in a steady-state en359 vironment. If, instead, we would consider a fluctuating environment in which, for instance, 360 the substrate source is only available for a finite time $T$, then the variation of the flux $J^{\mathcal{P}}(t)$ 361 would become relevant. The total amount of product formed during this time, $\mathcal{P}_{T}$, would 362 be

$$
\mathcal{P}_{T}=\int_{0}^{T} J^{\mathcal{P}}(t) \mathrm{d} t=\left\langle J^{\mathcal{P}}\right\rangle T+\int_{0}^{T} \delta J^{\mathcal{P}}(t) \mathrm{d} t,
$$

363 where $\delta J^{\mathcal{P}}(t)=J^{\mathcal{P}}(t)-\left\langle J^{\mathcal{P}}\right\rangle$ is the time-dependent fluctuation of the flux around its mean 364 value $\left\langle J^{\mathcal{P}}\right\rangle$, caused by the stochasticity of the enzymatic reaction and substrate diffusion. ${ }_{365}$ The integral over $\delta J^{\mathcal{P}}(t)$ scales as $\sqrt{T}$, due to the central limit theorem, such that its contri366 bution to $\mathcal{P}_{T}$ becomes negligible in the long time limit. However, for times $T$ comparable to 367 the intrinsic time-scales of the system (associated with the processes of diffusion, reaction, 368 and decay), the contribution of the fluctuations could become sizeable. In such scenarios, 369 a generalization of Markovitz' returns-variance analysis would be more appropriate than an 370 optimization of $\left\langle J^{\mathcal{P}}\right\rangle$. 


\section{$371 \quad$ Additive construction of optimal enzyme arrangements}

372 The above geometrical analysis leads us to an additive construction scheme, which gen373 erates the trajectory of optimal enzyme configurations as enzymes are added to the system. ${ }_{374}$ Generalizing from the example of Fig. 4, we consider a system in which space is discretized 375 into $N$ sites, denoting by $e_{i}=e\left(\mathbf{r}_{i}\right)$ the density of enzymes at position $\mathbf{r}_{i}$ and by $\rho_{i}=\rho\left(\mathbf{r}_{i}\right)$ the 376 corresponding substrate density. For a given total enzyme level $E_{T}$, there will be an optimal 377 enzyme configuration $\mathbf{e}^{*}=\left(e_{1}^{*}, e_{2}^{*}, \ldots, e_{N}^{*}\right)$ that maximizes $J^{\mathcal{P}}$ on the $(N-1)$-dimensional 378 hyperplane defined by $E_{T}=\sum_{i=1}^{N} e_{i}$. As in Fig. 4, the optimal configurations at different $E_{T}$ 379 values trace out a trajectory $\mathbf{e}^{*}\left(E_{T}\right)$ in $\mathbf{e}$ space. We seek a procedure to construct $\mathbf{e}^{*}\left(E_{T}\right)$. 380 We begin with no enzymes and iteratively add enzymes until the target $E_{T}$ value is reached. 381 According to the principle of homogeneous marginal returns, the largest components of $\frac{d J^{\mathcal{P}}}{d \mathbf{e}}$ 382 define the sites at which enzymes should be added in the next step. Addition may initially 383 be restricted to a single site, with further "construction sites" being introduced gradually as 384 different $E_{T}$ thresholds are crossed.

385 Since enzymes should be added at all positions where the marginal returns take on their 386 maximal value, we must determine how to partition new enzymes between these positions. 387 This partitioning corresponds to the tangent vector $\frac{d \mathbf{e}^{*}}{d E_{T}}$ of the optimal trajectory, which 388 can be obtained from the variation of the marginal returns as enzymes are added (see 389 Appendix G1),

$$
\frac{d}{d E_{T}} \frac{d J^{\mathcal{P}}}{d \mathbf{e}}=\mathbf{H} \frac{d \mathbf{e}^{*}}{d E_{T}} .
$$

390 Here, derivatives $\frac{d}{d E_{T}}$ are taken tangentially to the line of optimal enzyme configurations 391 and $\mathbf{H}$ is the $N \times N$ Hessian matrix of $J^{\mathcal{P}}$ in the space of enzyme densities, $H_{i j}=\frac{d^{2} J^{\mathcal{P}}}{d e_{i} d e_{j}}$. In 392 the subspace of only those sites where enzymes should be added ( 393 the left hand side of Eq. 12 becomes

$$
\frac{d}{d E_{T}} \frac{d J^{\mathcal{P}}}{d \mathbf{e}_{(n)}}=\frac{d \lambda_{e}}{d E_{T}} \mathbf{1}_{(n)},
$$

394 where the subscript $(n)$ indicates that we are considering only the $n$-dimensional subspace 395 of sites with equal returns, yielding

$$
\mathbf{H}_{(n)} \frac{d \mathbf{e}_{(n)}^{*}}{d E_{T}}=\frac{d \lambda_{e}}{d E_{T}} \mathbf{1}_{(n)} .
$$

396 This $n$-dimensional linear system can be solved for the tangent $\frac{d \mathbf{e}_{(n)}^{*}}{d E_{T}}$ to the optimal trajectory 
397 [47]. Taken together, we obtain the following additive construction scheme for the optimal 398 enzyme arrangement: 410 can be chosen using a criterion based on Eq. 10 (see Appendix G 2).

$411 \quad$ Fig. 5 illustrates the algorithm for a two-dimensional system featuring multiple sources of 412 substrate (dashed rectangles) with uniform influx on their surfaces and an absorbing outer 413 boundary. Fig. 5A compares two optimal enzyme configurations with a different amount of 414 enzymes. For low $E_{T}$ values, enzymes localize non-uniformly at the boundaries of the sources. ${ }_{415}$ For higher $E_{T}$ values, the optimal enzyme profile extends into the interior of the system. ${ }_{416}$ However, the highest densities of enzymes are found in the regions between the sources and 417 the outer boundary of the system, where the gradient of substrate concentration is steepest. ${ }_{418}$ A significant fraction of enzymes are thus devoted to substrates that, by the nature of where ${ }_{419}$ they are produced, are likely to rapidly diffuse out of the system.

420 Remarkably, the sites with the highest enzyme densities at small $E_{T}$ do not necessarily ${ }_{421}$ retain the highest densities as $E_{T}$ is increased. Fig. 5B compares the enzyme densities as a 422 function of $E_{T}$ at three positions (marked by colored squares in Fig. 5A). The blue location 423 is the first at which enzymes are added. However, although enzymes are added at the green 424 and red positions only later, the densities here become higher. Fig. 5C shows the respective 
A
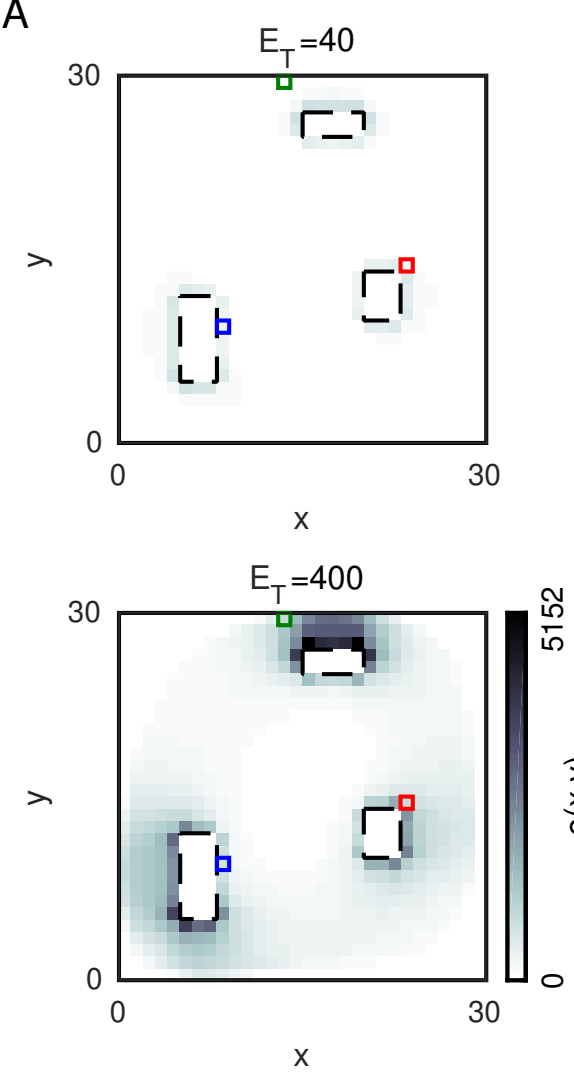

B
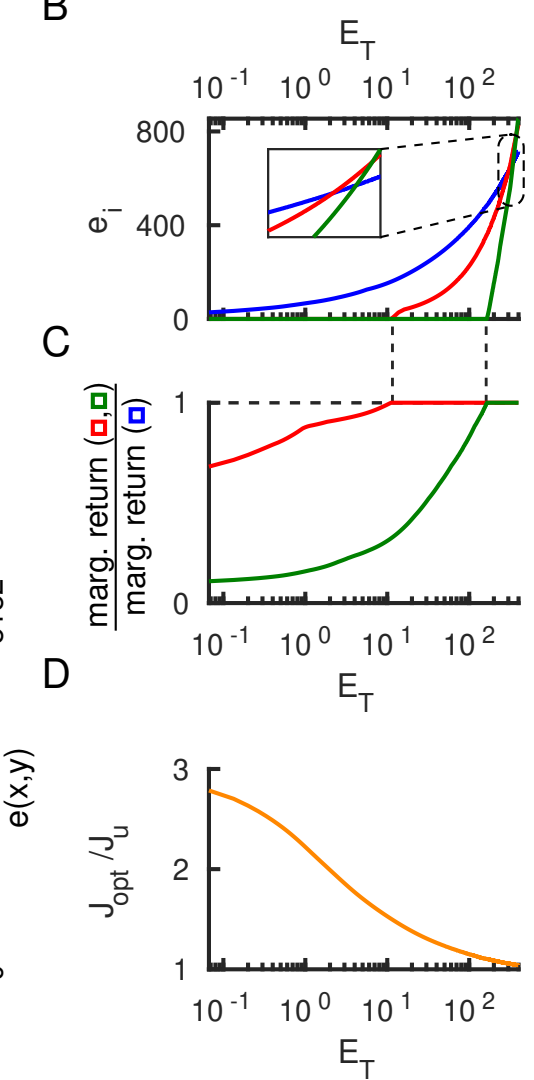

Figure 5. Optimal enzyme arrangements in a 2-dimensional system with multiple sources of substrate (dashed rectangles), calculated using the construction algorithm. (A) Snapshots of the optimal enzyme distribution at two $E_{T}$ levels. (B) Optimal enzyme densities and (C) ratios of marginal returns at the positions indicated by colored squares in (A). (D) The optimized reaction flux $J_{\mathrm{opt}}$ relative to the flux $J_{\mathrm{u}}$ produced by uniformly distributed enzymes, as a function of $E_{T}$.

${ }_{425}$ ratios of the returns of the red and green position to the returns from the blue position. 426 Enzymes are added at the green and red position only when the returns are equal and the ${ }_{427}$ ratios are one. At low enzyme amounts, the reaction flux of the optimal configuration is 428 about 3-fold higher than for the uniform configuration, whereas this advantage gradually 429 decreases for higher amounts of enzyme (Fig. 5D).

430 Compared to the stochastic search algorithms that have previously been used to find 431 the optimal enzyme arrangements in systems with simple geometries $[17,18]$, the additive 432 construction scheme derived here has several advantages. In terms of computational effort, it 433 generally performs better than stochastic optimization (see Appendix G 3), in particular for ${ }_{434}$ more complex systems. Moreover, while stochastic optimization proceeds by trial-and-error 
435 and can get stuck in local optima, the construction scheme follows a rational principle. By 436 design, the construction scheme not only generates the optimal enzyme arrangement for the 437 given total enzyme amount $E_{T}$, but all optimal configurations for total enzyme amounts up 438 to this level. As seen in the example of Fig. 5, this trajectory of optimal configurations leads 439 to additional insight about the properties of the particular system at hand.

440 The construction scheme can also be adapted to other allocation problems in which 441 investments and returns are coupled. As long as the resources to be invested follow a linear 442 budget constraint, the adaptation amounts to a modification of the objective function. For 443 instance, as mentioned above, one could consider an objective function penalizing high 444 variance as in Markovitz' problem [11]. One could also consider problems where the capital 445 of the investor grows exponentially (iterative reinvestment) as in Kelly's problem [10] or 446 more complex variants [48]. In each case, one needs to compute the marginal returns and the ${ }_{447}$ Hessian of the new objective function with respect to the resources invested in the different 448 assets, and determine the optimal allocation of resources as the total budget gradually 449 increases via the construction scheme. In Appendix H, we present a generalization of Kelly's 450 problem to a case with coupled investments and returns for which the objective function 451 becomes the long-term capital growth rate and the above additive construction scheme can 452 be used.

\section{DISCUSSION}

${ }_{454}$ We presented a solution for the problem of optimally allocating enzymes in space, to 455 maximize the reaction flux of an enzymatic reaction. Our solution encompasses a class of 456 systems with potentially complex geometries, in which the substrate enters via internal or ${ }_{457}$ external boundaries, is transported via diffusion and possibly advection, and can be lost by 458 leakage or competing reactions (Fig. 1). The solution is based on the concept of a 'marginal 459 returns landscape' (Fig. 2), defined as the derivative $\frac{d J^{\mathcal{P}}}{d e(\mathbf{r})}$ of the total reaction flux with 460 respect to the local enzyme density (Eq. 7). The optimal arrangement of enzymes is such 461 that the marginal returns are spatially homogeneous over all positions with enzymes. This 462 criterion of homogeneous marginal returns (HMR) can more generally be applied to betting 463 games where each bet globally feeds back onto all returns (Appendix H). In the enzyme 464 allocation problem, the feedback is generated by enzymes locally depleting their substrate, 
465 which affects the global substrate profile.

${ }_{466}$ The HMR criterion leads to a general local condition for the occurrence of a localization467 delocalization transition in the optimal enzyme arrangement (Eq. 10), which compares the 468 local diffusive flux $j^{D}(\mathbf{s})$ to the local reaction flux $j^{\mathcal{P}}(\mathbf{s})$ at a source position $\mathbf{s}$ : As long 469 as $j^{D}(\mathbf{s})>j^{\mathcal{P}}(\mathbf{s})$, localization of enzymes at the source is optimal, while the two fluxes 470 are equal at the transition point and above (Fig. 3). The simplicity of this flux condition 471 makes it practical for application to experimental systems. For instance, for an in vivo 472 system in which enzyme localization is observed and the enzyme abundance is quantified, 473 the condition can be used to estimate whether the observed enzyme localization indeed 474 maximizes the reaction flux, or whether it is more likely to play a regulatory role $[7,8]$. In 475 this context, it is beneficial that the flux condition is not affected by any advective transport 476 of the substrate, which may be present in the system, e.g. due to cytoplasmic streaming 477 or transport by molecular motors. This independence is due to the deterministic nature of 478 advective transport - the HMR criterion is fundamentally a bet-hedging strategy to deal 479 with the probabilistic nature of diffusive transport.

480 Just as the Kelly criterion [10] and returns-variance analysis [11] provide rules for op481 timally placing bets or optimally constructing portfolios, the HMR criterion allowed us to 482 derive an algorithm for constructing optimal enzyme arrangements (Figs. 4 and 5). This 483 algorithm is obtained by analyzing how the marginal returns change at each position as 484 the amount of enzymes in the system is increased (Eq. 14). It constitutes an exact deter485 ministic construction scheme, which is applicable to systems with arbitrary geometries and 486 multiple sources. Here, we presented the simplest general form of the algorithm. Various 487 modifications can be made to improve its efficiency for particular systems. Using the sys488 tem geometry as a guide, one can limit the sites at which the marginal returns need to be 489 evaluated in each update step. For example, at low $E_{T}$ values the positions with the highest 490 returns coincide with sources of $\mathcal{S}$. Generally it suffices to compute the marginal returns 491 only at source positions, sites where enzymes are already present, and neighboring sites. One 492 could further speed up the algorithm by enabling larger increments $\delta E_{T}$ with higher-order 493 update schemes for $\mathbf{e}^{*}$.

${ }_{494}$ We explicitly considered absorbing and reflective boundaries, also allowing for non495 uniform and discontinuous influx profiles. However our results can be extended to systems 496 with partially permeable boundaries, corresponding to $k(\mathbf{s})=1$ and $h(\mathbf{s})=p(\mathbf{s})>0$ in 
${ }_{497}$ Eq. 3, where $p(\mathbf{s})$ is the permeability. For such systems, the HMR criterion still holds. 498 Similarly, the condition of Eq. 9 still determines transitions in the optimal enzyme profile. 499 However, expressing this condition in term of substrate fluxes will lead to a more complicated 500 expression than Eq. 10. A permeable boundary typically increases the transition threshold ${ }_{501} E_{T}^{t}$. For example, in the one-dimensional system of Fig. 2, a non-zero permeability at the 502 origin shifts the threshold value of $E_{T}$ from $\alpha^{-1}$ to $\alpha^{-1}(1+p(0) L / D)$.

503 Our continuous reaction-diffusion model neglects the finite size of enzyme molecules that 504 limits the attainable enzyme density. Imposing a maximal density condition $e(\mathbf{r}) \leq e_{\max }$ 505 at each point [16] could be incorporated into our analytical framework as an additional 506 constraint in the Lagrangian. This would introduce an effective position-dependent cost 507 in Eq. 7, resulting in a marginal returns landscape that is a function of position. The 508 construction algorithm could still be employed with the additional condition that enzymes 509 cannot be added at positions where $e_{i}=e_{\max }$, such that Eq. 14 would be restricted to 510 positions with sub-maximal enzyme densities.

511 The construction algorithm for optimal enzyme arrangements appears suited for appli512 cations in synthetic biology [19]. The attachment of enzymes to membranes can be con513 trolled by fusing enzymes to transmembrane proteins $[49,50]$ or by using protein scaffolds $514[51,52]$. The arrangement of enzymes in the interior of membrane-enclosed systems can 515 be partially controlled with RNA assemblies [53], synthetic protein scaffolds [20], or fusion 516 proteins [16]. Enzyme positioning on surfaces can be controlled with nanometer precision 517 via single-molecule cut-and-paste surface assembly [54, 55], and in 3D by arranging enzymes 518 on DNA scaffolds [56-59]. Combining this arsenal of techniques with the rational construc519 tion scheme, one could optimize synthetic bioreactors to produce drug molecules [20-22] or 520 biofuels [23, 24].

\section{$521 \quad$ MATERIALS AND METHODS}

\section{Computation of $\frac{d J}{d \mathbf{e}}$ and $\mathbf{H}$ for linear reactions}

${ }_{523}$ For a discrete reaction-diffusion system with linear reactions, following the dynamics of ${ }_{524}$ Eq. 1, the steady-state equation can be written in matrix form as

$$
(\mathbf{D}+\mathbf{V}) \boldsymbol{\rho}-\alpha \mathbf{e} \odot \boldsymbol{\rho}-\sigma \boldsymbol{\rho}=\mathbf{A}
$$




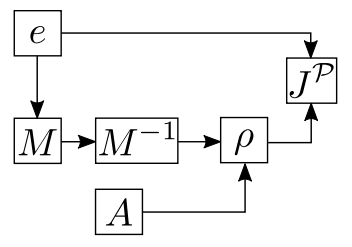

Figure 6. Dependency graph for a discrete reaction-diffusion system with linear reaction kinetics.

${ }_{525}$ where $\mathbf{D}$ and $\mathbf{V}$ are the diffusion and advection operator on the lattice respectively, $\boldsymbol{\rho}$ ${ }_{526}$ the substrate density and $\mathbf{A}$ the substrate influx vector, while $\odot$ denotes the element-wise ${ }_{527}$ (Hadamard) product. The reaction flux $J^{\mathcal{P}}$ is given by the scalar product $J^{\mathcal{P}}=\alpha \mathbf{e} \cdot \boldsymbol{\rho}$. ${ }_{528}$ Thus e enters $J^{\mathcal{P}}$ both directly and through the solution $\boldsymbol{\rho}$ of Eq. 15 . These multiple 529 dependencies of $J^{\mathcal{P}}$ on e are summarized by the dependency graph in Fig. 6, where the 530 matrix $\mathbf{M}=\mathbf{D}+\mathbf{V}-\alpha \operatorname{diag}(\mathbf{e})-\sigma \operatorname{diag}(\mathbf{1})$ is the operator that applied to $\boldsymbol{\rho}$ gives the 531 substrate source vector $\mathbf{M} \boldsymbol{\rho}=\mathbf{A}$, $\operatorname{diag}(\mathbf{e})$ is the diagonal matrix with diagonal elements 532 given by $\mathbf{e}$ and $\operatorname{diag}(\mathbf{1})$ is the identity matrix.

The marginal returns vector $\frac{d J^{\mathcal{P}}}{d \mathbf{e}}$ can be evaluated by back-propagating the derivative of the reaction flux with respect to the enzyme vector through the dependency graph. We find that

$$
\begin{aligned}
\frac{d J^{\mathcal{P}}}{d \mathbf{e}} & =\frac{\partial J^{\mathcal{P}}}{\partial \mathbf{e}}+\left(\frac{d \boldsymbol{\rho}}{d \mathbf{e}}\right)^{t} \frac{\partial J^{\mathcal{P}}}{\partial \boldsymbol{\rho}} \\
& =\frac{\partial J^{\mathcal{P}}}{\partial \mathbf{e}}+\left(\frac{d\left(\mathbf{M}^{-1} \mathbf{A}\right)}{d \mathbf{M}} \frac{d \mathbf{M}}{d \mathbf{e}}\right)^{t} \frac{\partial J^{\mathcal{P}}}{\partial \boldsymbol{\rho}}
\end{aligned}
$$

533 where $\frac{d \boldsymbol{\rho}}{d \mathbf{e}}$ is a matrix with $(i, j)^{\text {th }}$ element given by $\frac{d \rho_{i}}{d e_{j}}$, and ${ }^{t}$ denotes the transpose. Each 534 of the derivatives appearing in Eq. 17 has a simple form, which we substitute to obtain the 535 marginal returns vector,

$$
\frac{d J^{\mathcal{P}}}{d \mathbf{e}}=\alpha \boldsymbol{\rho} \odot\left[\mathbf{1}+\alpha\left(\mathbf{M}^{-1}\right)^{t} \mathbf{e}\right]
$$

536 where $\mathbf{1}$ is a vector of ones.

${ }_{537}$ By taking a second derivative with respect to e, and again by back-propagating the 538 derivatives, we can determine the Hessian,

$$
\begin{aligned}
\mathbf{H}=\alpha^{2} & \left\{\left[\mathbf{M}^{-1} \odot\left(\left\{\mathbf{1}+\alpha\left(\mathbf{M}^{-1}\right)^{t} \mathbf{e}\right\} \otimes \boldsymbol{\rho}\right)\right]\right. \\
& \left.+\left[\mathbf{M}^{-1} \odot\left(\left\{\mathbf{1}+\alpha\left(\mathbf{M}^{-1}\right)^{t} \mathbf{e}\right\} \otimes \boldsymbol{\rho}\right)\right]^{t}\right\},
\end{aligned}
$$

539 where $\otimes$ indicates the outer product. 


\section{$540 \quad$ Non-linear reactions}

541 For non-linear reaction kinetics the reaction-diffusion equation becomes

$$
(\mathbf{D}+\mathbf{V}) \boldsymbol{\rho}-\alpha \mathbf{e} \odot \mathbf{F}-\sigma \boldsymbol{\rho}=\mathbf{A}
$$

${ }_{542}$ where $\mathbf{F}$ is the vector with $F_{i}=F\left[\rho_{i}\right]$. Since $F[\rho]$ is non-linear, it is no longer possible 543 to write the reaction-diffusion equation as a linear system that can be solved for $\boldsymbol{\rho}$. As 544 a result the dependency graph includes a loop, making it inconvenient to back-propagate 545 derivatives. Instead, taking derivatives of the reaction-diffusion equation with respect to e, 546 we can directly solve for

$$
\frac{d \boldsymbol{\rho}}{d \mathbf{e}}=\alpha \mathbf{N}^{-1} \operatorname{diag}(\mathbf{F})
$$

${ }_{547}$ where $\mathbf{N}=\mathbf{D}+\mathbf{V}-\alpha \operatorname{diag}\left(\mathbf{e} \odot \mathbf{F}^{\prime}\right)-\sigma \operatorname{diag}(\mathbf{1})$ and $F_{i}^{\prime}=F^{\prime}\left[\rho_{i}\right]$. We then take derivatives ${ }_{548}$ of $J^{\mathcal{P}}=\alpha \mathbf{e} \cdot \mathbf{F}$ with respect to $\mathbf{e}$, to obtain the marginal returns vector,

$$
\begin{aligned}
\frac{d J^{\mathcal{P}}}{d \mathbf{e}} & =\alpha\left[\mathbf{F}+\left(\frac{d \boldsymbol{\rho}}{d \mathbf{e}}\right)^{t}\left\{\mathbf{e} \odot \mathbf{F}^{\prime}\right\}\right] \\
& =\alpha \mathbf{F} \odot\left[\mathbf{1}+\alpha\left(\mathbf{N}^{-1}\right)^{t}\left\{\mathbf{e} \odot \mathbf{F}^{\prime}\right\}\right] .
\end{aligned}
$$

${ }_{549}$ By taking a further derivative of the returns vector with respect to e we find the components 550 of the Hessian,

$$
\begin{aligned}
& \mathbf{H}_{j, k}=\alpha^{2}\left\{\mathbf{N}_{j, k}^{-1}\left[\mathbf{F} \otimes\left(\mathbf{F}^{\prime}+\alpha\left(\mathbf{N}^{-1}\right)^{t}\left\{\mathbf{e} \odot \mathbf{F}^{2}\right\}\right)\right]_{k, j}\right. \\
& \left.+\mathbf{N}_{k, j}^{-1}\left[\mathbf{F} \otimes\left(\mathbf{F}^{\prime}+\alpha\left(\mathbf{N}^{-1}\right)^{t}\left\{\mathbf{e} \odot \mathbf{F}^{\prime 2}\right\}\right)\right]_{j, k}\right\} \\
& +\alpha^{3} F_{j} F_{k} \sum_{i} \mathbf{N}_{i, j}^{-1} e_{i} F_{i}^{\prime \prime} \mathbf{N}_{i, k}^{-1}\left[1+\alpha\left[\left(\mathbf{N}^{-1}\right)^{t}\left\{\mathbf{e} \odot \mathbf{F}^{\prime}\right\}\right]_{i}\right],
\end{aligned}
$$

551 which simplifies to Eq. 19 for linear reactions with $F^{\prime \prime}=0$.

\section{ACKNOWLEDGMENTS}

${ }_{553}$ This work was supported by the German Research Foundation via SFB1032 "Nanoagents ${ }_{554}$ for Spatiotemporal Control of Molecular and Cellular Reactions" and the excellence clus555 ter ORIGINS. G.G. was supported by a DFG Fellowship through the Graduate School of 556 Quantitative Biosciences Munich (QBM). 


\section{${ }_{557}$ AUTHOR CONTRIBUTIONS}

${ }_{558}$ All authors designed research and developed the theoretical framework. G.G. and F.T. 559 developed computational methods and analyzed data. G.G., F.T., and U.G. wrote the 560 paper.

575 [10] J. L. Kelly, Bell Labs Tech. J., 35, 917 (1956).

576 [11] H. Markowitz, J. Finance, 7, 77 (1952).

577 [12] E. O. Thorp, in Stochastic Optimization Models in Finance (Elsevier, 1975) pp. 599-619.

578 [13] D. Cohen, Journal of Theoretical Biology, 12, 119 (1966), ISSN 0022-5193.

579 [14] P. Bauler, G. Huber, T. Leyh, and J. A. McCammon, J. Phys. Chem. Lett., 1, 1332 (2010).

580 [15] O. Idan and H. Hess, ACS Nano, 7, 8658 (2013).

581 [16] M. Castellana, M. Z. Wilson, Y. Xu, P. Joshi, I. M. Cristea, J. D. Rabinowitz, Z. Gitai, and $582 \quad$ N. S. Wingreen, Nat. Biotechnol., 32, 1011-1018 (2014).

583 [17] A. Buchner, F. Tostevin, and U. Gerland, Phys. Rev. Lett., 110, 208104 (2013).

584 [18] A. Buchner, F. Tostevin, F. Hinzpeter, and U. Gerland, J. Chem. Phys., 139, 135101 (2013). 
585 [19] C. M. Agapakis, P. M. Boyle, and P. A. Silver, Nat. Chem. Biol., 8, 527 (2012).

586 [20] J. E. Dueber, G. C. Wu, G. R. Malmirchegini, T. S. Moon, C. J. Petzold, A. V. Ullal, K. L.

587 Prather, and J. D. Keasling, Nat. Biotechnol., 27, 753 (2009).

588 [21] J. Zhou and B. Xu, Bioconjugate Chem., 26, 987 (2015).

589 [22] J. Li, Y. Kuang, J. Shi, J. Zhou, J. E. Medina, R. Zhou, D. Yuan, C. Yang, H. Wang, Z. Yang, $590 \quad$ et al., Angewandte Chemie, 127, 13505 (2015).

591 [23] S. Moraïs, Y. Barak, J. Caspi, Y. Hadar, R. Lamed, Y. Shoham, D. B. Wilson, and E. A. $592 \quad$ Bayer, Appl. Environ. Microbiol., 76, 3787 (2010).

593 [24] S.-L. Tsai, J. Oh, S. Singh, R. Chen, and W. Chen, Appl. Environ. Microbiol., 75, 6087 (2009).

594 [25] E. Kussell and S. Leibler, Science, 309, 2075 (2005), ISSN 0036-8075, 1095-9203.

595 [26] O. Rivoire and S. Leibler, J. Stat. Phys., 142, 1124 (2011), ISSN 1572-9613.

596 [27] G. Fritz, N. Walker, and U. Gerland, J. Mol. Biol., 431, 4760 (2019).

597 [28] T. Taillefumier, A. Posfai, Y. Meir, and N. S. Wingreen, Elife, 6, e22644 (2017).

598 [29] O. Shoval, H. Sheftel, G. Shinar, Y. Hart, O. Ramote, A. Mayo, E. Dekel, K. Kavanagh, and $599 \quad$ U. Alon, Science, 336, 1157 (2012), ISSN 0036-8075, 1095-9203.

600 [30] R. E. Goldstein and J.-W. van de Meent, Interface Focus, 5, 20150030 (2015).

601 [31] J. F. Presley, N. B. Cole, T. A. Schroer, K. Hirschberg, K. J. Zaal, and J. Lippincott-Schwartz, $602 \quad$ Nature, $\mathbf{3 8 9}, 81(1997)$.

603 [32] B. Hove-Jensen, K. R. Andersen, M. Kilstrup, J. Martinussen, R. L. Switzer, and M. Wille604 moës, Microbiol. Mol. Biol. Rev., 81, e00040 (2017).

605 [33] E. W. Miles, S. Rhee, and D. R. Davies, J. Biol. Chem., 274, 12193 (1999).

606 [34] J. R. Robbins, D. Monack, S. J. McCallum, A. Vegas, E. Pham, M. B. Goldberg, and J. A. 607 Theriot, Mol. Microbiol., 41, 861 (2001).

608 [35] Y. E. Chen, C. Tropini, K. Jonas, C. G. Tsokos, K. C. Huang, and M. T. Laub, Proc. Natl. 609 Acad. Sci. U.S.A., 108, 1052 (2011).

610 [36] T. E. Saunders, K. Z. Pan, A. Angel, Y. Guan, J. V. Shah, M. Howard, and F. Chang, Dev. 611 Cell, 22, 558 (2012).

612 [37] D. Oh, C.-H. Yu, and D. J. Needleman, Proc. Natl. Acad. Sci. U.S.A., 113, 8729 (2016).

613 [38] G. Hrazdina and G. J. Wagner, Arch. Biochem. Biophys., 237, 88 (1985).

${ }_{614}[39]$ J. W. Graham, T. C. Williams, M. Morgan, A. R. Fernie, R. G. Ratcliffe, and L. J. Sweetlove, $615 \quad$ Plant Cell, 19, 3723 (2007). 
${ }_{616}[40]$ J. B. French, S. A. Jones, H. Deng, A. M. Pedley, D. Kim, C. Y. Chan, H. Hu, R. J. Pugh, 617 H. Zhao, Y. Zhang, et al., Science, 351, 733 (2016).

${ }_{618}[41]$ T. Laursen, J. Borch, C. Knudsen, K. Bavishi, F. Torta, H. J. Martens, D. Silvestro, N. S. 619 Hatzakis, M. R. Wenk, T. R. Dafforn, et al., Science, 354, 890 (2016).

620 [42] M. E. Campanella, H. Chu, and P. S. Low, Proc. Natl. Acad. Sci. U.S.A., 102, 2402 (2005).

${ }_{621}[43]$ H. W. Kuhn and A. W. Tucker, Proceedings of the Second Berkeley Symposium on Mathe622 matical Statistics and Probability, 481 (1951), publisher: University of California Press.

623 [44] W. Karush, M. Sc. Dissertation. Dept. of Mathematics, Univ. of Chicago (1939).

${ }_{624}$ [45] A. Bar-Even, E. Noor, Y. Savir, W. Liebermeister, D. Davidi, D. S. Tawfik, and R. Milo, 625 Biochemistry, 50, 4402 (2011), ISSN 0006-2960.

626 [46] B. Ho, A. Baryshnikova, and G. W. Brown, Cell Syst., 6, 192 (2018).

627 [47] In general we do not know $\frac{d \lambda_{e}}{d E_{T}}$ a priori. However, this scalar prefactor affects only the length, and not the direction, of $\frac{d \mathbf{e}^{*}}{d E_{T}}$. Hence it suffices to treat $\frac{d \lambda_{e}}{d E_{T}}$ as an arbitrary constant, and 629 subsequently to rescale the resulting $\frac{d \mathbf{e}^{*}}{d E_{T}}$ to unit length once its direction is known.

630 [48] L. Dinis, J. Unterberger, and D. Lacoste, EPL (Europhysics Letters), 131, 60005 (2020), ISSN $631 \quad 0295-5075$.

632 [49] T. Thomik, I. Wittig, J.-y. Choe, E. Boles, and M. Oreb, Nat. Chem. Biol., 13, 1158 (2017).

${ }_{633}$ [50] H. Rabeharindranto, S. Castaño-Cerezo, T. Lautier, L. F. Garcia-Alles, C. Treitz, A. Tholey, $634 \quad$ and G. Truan, Metab. Eng. Commun., 8, e00086 (2019).

635 [51] S.-L. Tsai, N. A. DaSilva, and W. Chen, ACS Synth. Biol., 2, 14 (2013).

636 [52] J.-L. Lin, J. Zhu, and I. Wheeldon, ACS Synth. Biol., 6, 1534 (2017).

637 [53] C. J. Delebecque, A. B. Lindner, P. A. Silver, and F. A. Aldaye, Science, 333, 470 (2011).

638 [54] S. Kufer, E. Puchner, H. Gumpp, T. Liedl, and H. Gaub, Science, 319, 594 (2008).

639 [55] K. R. Erlich, S. M. Sedlak, M. A. Jobst, L. F. Milles, and H. E. Gaub, Nanoscale, 11, 407 640 (2019).

${ }_{641}$ [56] J. Müller and C. M. Niemeyer, Biochem. Biophys. Res. Commun., 377, 62 (2008).

642 [57] O. I. Wilner, Y. Weizmann, R. Gill, O. Lioubashevski, R. Freeman, and I. Willner, Nat. $643 \quad$ Nanotechnol., 4, 249 (2009).

644 [58] J. Fu, M. Liu, Y. Liu, N. W. Woodbury, and H. Yan, J. Am. Chem. Soc., 134, 5516 (2012).

${ }_{645}^{6}$ [59] J. Fu, Y. R. Yang, A. Johnson-Buck, M. Liu, Y. Liu, N. G. Walter, N. W. Woodbury, and 646 H. Yan, Nat. Nanotechnol., 9, 531 (2014). 


\section{Appendix A: General Lagrangian with multiple subspaces}

${ }_{648}$ As noted in the main text, our functional Lagrangian formalism assumes smooth, positive 649 enzyme distributions within different subdomains. At the interfaces of such domains, the 650 optimal enzyme distributions may feature discontinuities. For example, there may be a finite 651 enzyme density in one region of the system, and no enzymes (zero density) in another region. 652 These subdomains should be picked in the proximities of the substrate sources. For example 653 if we have patches of sources on the surface domains, for each source patch we would consider 654 a surface subdomain around the patch and a correspondent volume subdomain in proximity 655 of the source. These are the regions where it is optimal to distribute the enzymes as diffusion 656 does not allow for peak of substrate concentration away from the sources. Within each such 657 subdomain $i$, the substrate and enzyme densities $\rho^{(i)}(\mathbf{r})$ and $e^{(i)}(\mathbf{r})$ are smooth and their 658 local gradients are well defined. However, at the interfaces between such subdomains, $e(\mathbf{r})$ 659 need not be continuous (although $\rho(\mathbf{r})$ still must be). The positions of the interfaces, and 660 therefore the extents of the different domains, should be optimized over and as we have seen 661 in the main text they depend on the $E_{T}$ considered.

${ }_{662}$ In this formulation, the total reaction flux can be written as a sum of contributions from ${ }_{663}$ each surface and boundary domain, and the corresponding reaction-diffusion or boundary ${ }_{664}$ condition acts as a constraint that must be satisfied within each domain. In addition, we ${ }_{665}$ introduce explicit matching conditions on $\rho(\mathbf{r})$ at the interfaces $I$. The resulting Lagrangian 666 has the following form:

$$
\begin{aligned}
\mathcal{L}= & \sum_{i} \int_{S_{i}} k_{\mathrm{cat}} e_{S}^{(i)}(\mathbf{s}) F\left[\rho^{(i)}(\mathbf{s})\right] \mathrm{d} \mathbf{s}+\sum_{j} \int_{V_{j}} k_{\mathrm{cat}} e^{(j)}(\mathbf{r}) F\left[\rho^{(j)}(\mathbf{r})\right] \mathrm{d} \mathbf{r} \\
& -\lambda_{e}\left[\sum_{i} \int_{S_{i}} e_{S}^{(i)}(\mathbf{s}) \mathrm{d} \mathbf{s}+\sum_{j} \int_{V_{j}} e^{(j)}(\mathbf{r}) \mathrm{d} \mathbf{r}-E_{T}\right] \\
& +\sum_{j} \int_{V_{j}} \lambda_{V}^{(j)}(\mathbf{r})\left\{D \nabla^{2} \rho^{(j)}(\mathbf{r})-\nabla \cdot\left[\rho^{(j)}(\mathbf{r}) \mathbf{v}(\mathbf{r})\right]-\sigma \rho^{(j)}(\mathbf{r})-k_{\mathrm{cat}} e^{(j)}(\mathbf{r}) F\left[\rho^{(j)}(\mathbf{r})\right]\right\} \mathrm{d} \mathbf{r} \\
& +\sum_{i} \int_{S_{i}} \lambda_{S}^{(i)}(\mathbf{s})\left\{k(\mathbf{s})\left[j(\mathbf{s})-D \frac{\partial \rho^{(l)}}{\partial \mathbf{n}(\mathbf{s})}+\rho^{(l)}(\mathbf{s}) \mathbf{v}(\mathbf{s}) \cdot \hat{\mathbf{n}}(\mathbf{s})-k_{\mathrm{cat}} e_{S}^{(i)}(\mathbf{s}) F\left[\rho^{(l)}(\mathbf{s})\right]\right]-h(\mathbf{s}) \rho^{(l)}(\mathbf{s})\right\} \mathrm{d} \mathbf{s} \\
& +\sum_{k} \int_{I_{k}}\left\{\lambda_{I}^{(k)}(\mathbf{r})\left[\rho^{\left(k_{1}\right)}(\mathbf{r})-\rho^{\left(k_{2}\right)}(\mathbf{r})\right]+\lambda_{I}^{(k)}(\mathbf{r}) \cdot\left[\nabla \rho^{\left(k_{1}\right)}(\mathbf{r})-\nabla \rho^{\left(k_{2}\right)}(\mathbf{r})\right]\right\} \mathrm{d} \mathbf{r} .
\end{aligned}
$$

${ }_{667}$ where $l$ represents the index of the volume domain adjacent to the boundary at $\mathbf{s}$, and $k_{1}$, 
${ }_{668} k_{2}$ represent the two domains on either side of the interface $I_{k}$. Here $\lambda_{I}(\mathbf{r})$ is a Lagrange 669 multiplier that enforces smoothness of the density profile at each point $\mathbf{r}$ on an interface 670 between two domains, and $\lambda_{I}^{\prime}(\mathbf{r})$ is a vector of Lagrangian multipliers where each component 671 enforces smoothness of $\rho(\mathbf{r})$ along one of the system dimensions.

It is convenient to consider first some manipulations of the general Lagrangian, Eq. A1. Using the vector identity $\nabla \cdot(f \mathbf{a})=\nabla f \cdot \mathbf{a}+f \nabla \cdot \mathbf{a}$ we can rearrange the diffusive and advective transport terms to read

$$
\begin{aligned}
\lambda_{V}(\mathbf{r}) \nabla^{2} \rho(\mathbf{r}) & =\nabla \cdot\left[\lambda_{V}(\mathbf{r}) \nabla \rho(\mathbf{r})-\rho(\mathbf{r}) \nabla \lambda_{V}(\mathbf{r})\right]+\rho(\mathbf{r}) \nabla^{2} \lambda_{V}(\mathbf{r}), \\
\lambda_{V}(\mathbf{r}) \nabla \cdot[\rho(\mathbf{r}) \mathbf{v}(\mathbf{r})] & =\nabla \cdot\left[\rho(\mathbf{r}) \lambda_{V}(\mathbf{r}) \mathbf{v}(\mathbf{r})\right]-\rho(\mathbf{r}) \mathbf{v}(\mathbf{r}) \cdot \nabla \lambda_{V}(\mathbf{r})
\end{aligned}
$$

672 Applying Gauss theorem $\left(\int_{V} \nabla \cdot \mathbf{a} \mathrm{d} \mathbf{r}=\oint \mathbf{a} \cdot \hat{\mathbf{n}}(\mathbf{s}) \mathrm{d} \mathbf{s}\right)$, Eq. A1 becomes

$$
\begin{aligned}
\mathcal{L}= & \sum_{i} \int_{S_{i}} k_{\mathrm{cat}} e_{S}^{(i)}(\mathbf{s}) F\left[\rho^{(i)}(\mathbf{s})\right] \mathrm{d} \mathbf{s}+\sum_{j} \int_{V_{j}} k_{\mathrm{cat}} e^{(j)}(\mathbf{r}) F\left[\rho^{(j)}(\mathbf{r})\right] \mathrm{d} \mathbf{r} \\
& -\lambda_{e}\left[\sum_{i} \int_{S_{i}} e_{S}^{(i)}(\mathbf{s}) \mathrm{d} \mathbf{s}+\sum_{j} \int_{V_{j}} e^{(j)}(\mathbf{r}) \mathrm{d} \mathbf{r}-E_{T}\right] \\
& +\sum_{j} \int_{V_{j}}\left\{\rho^{(j)}(\mathbf{r})\left[D \nabla^{2} \lambda_{V}^{(j)}(\mathbf{r})+\mathbf{v}(\mathbf{r}) \cdot \nabla \lambda_{V}^{(j)}(\mathbf{r})-\sigma \lambda_{V}^{(j)}(\mathbf{r})\right]-k_{\mathrm{cat}} \lambda_{V}^{(j)}(\mathbf{r}) e^{(j)}(\mathbf{r}) F\left[\rho^{(j)}(\mathbf{r})\right]\right\} \mathrm{d} \mathbf{r} \\
& +\sum_{i} \int_{S_{i}} \lambda_{S}^{(i)}(\mathbf{s})\left\{k(\mathbf{s})\left[j(\mathbf{s})-D \frac{\partial \rho^{(l)}}{\partial \mathbf{n}(\mathbf{s})}+\rho^{(l)}(\mathbf{s}) \mathbf{v}(\mathbf{s}) \cdot \hat{\mathbf{n}}(\mathbf{s})-k_{\mathrm{cat}} e_{S}^{(i)}(\mathbf{s}) F\left[\rho^{(l)}(\mathbf{s})\right]\right]-h(\mathbf{s}) \rho^{(l)}(\mathbf{s})\right\} \mathrm{d} \mathbf{s} \\
& +\sum_{k} \int_{I_{k}}\left\{\lambda_{I}^{(k)}(\mathbf{r})\left[\rho^{\left(k_{1}\right)}(\mathbf{r})-\rho^{\left(k_{2}\right)}(\mathbf{r})\right]+\lambda_{I}^{(k)}(\mathbf{r}) \cdot\left[\nabla \rho^{\left(k_{1}\right)}(\mathbf{r})-\nabla \rho^{\left(k_{2}\right)}(\mathbf{r})\right]\right\} \mathrm{d} \mathbf{r} \\
& +\sum_{i} \int_{S_{i}}\left\{D \lambda_{V}^{(l)}(\mathbf{s}) \frac{\partial \rho^{(l)}}{\partial \mathbf{n}(\mathbf{s})}-D \rho^{(l)}(\mathbf{s}) \frac{\partial \lambda_{V}^{(l)}}{\partial \mathbf{n}(\mathbf{s})}-\lambda_{V}^{(l)}(\mathbf{s}) \rho^{(l)}(\mathbf{s}) \mathbf{v}(\mathbf{s}) \cdot \hat{\mathbf{n}}(\mathbf{s})\right\} \mathrm{d} \mathbf{s} \\
& +\sum_{k} \int_{I_{k}}\left\{D \lambda_{V}^{\left(k_{1}\right)}(\mathbf{r}) \frac{\partial \rho^{\left(k_{1}\right)}}{\partial \mathbf{n}(\mathbf{r})}-D \rho^{\left(k_{1}\right)}(\mathbf{r}) \frac{\partial \lambda_{V}^{\left(k_{1}\right)}}{\partial \mathbf{n}(\mathbf{r})}-D \lambda_{V}^{\left(k_{2}\right)}(\mathbf{r}) \frac{\partial \rho^{\left(k_{2}\right)}}{\partial \mathbf{n}(\mathbf{r})}+D \rho^{\left(k_{2}\right)}(\mathbf{r}) \frac{\partial \lambda_{V}^{\left(k_{2}\right)}}{\partial \mathbf{n}(\mathbf{r})}\right. \\
& \left.-\left[\lambda_{V}^{\left(k_{1}\right)}(\mathbf{r}) \rho^{\left(k_{1}\right)}(\mathbf{r})-\lambda_{V}^{\left(k_{2}\right)}(\mathbf{r}) \rho^{\left(k_{2}\right)}(\mathbf{r})\right] \mathbf{v}(\mathbf{r}) \cdot \hat{\mathbf{n}}(\mathbf{r})\right\} \mathrm{d} \mathbf{r}
\end{aligned}
$$

${ }_{673}$ Here the last three lines contain the contributions from the boundary integral.

\section{Appendix B: Optimal enzyme arrangement in a one-dimensional system}

675 In this section we go through in detail the full analytic optimization of the simplest 676 exactly-solvable model. We consider a one-dimensional system with a source of substrate at 
677 one boundary at $x=0,\left(j(0)=j_{0}, k(0)=1, h(0)=0\right)$ and an absorbing boundary at $x=L$ ${ }_{678}(j(L)=k(L)=0, h(L)=1)$. Further we assume a linear reaction functional, no drift and 679 no instability of $\mathcal{S}$, i.e. $F[\rho]=\rho / K_{\mathrm{M}}$ and $v=\sigma=0$.

680 In our formalism we divide the system into two boundary domains $\left(S_{1}: x=0\right.$ and $S_{2}$ : $\left.{ }_{681} x=L\right)$ and two "volume" domains $\left(V_{1}: 0 \leq x \leq x_{0}\right.$ and $V_{2}: x_{0} \leq x \leq L$, with $0 \leq x_{0} \leq L$ 682 a parameter to be optimized). For simplicity of presentation we will assume that enzymes ${ }_{683}$ can be present at $x=0$ or in the domain $V_{1}$, while the boundary at $x=L$ and the domain ${ }_{684} V_{2}$ are free of enzymes. Whilst one can include an arbitrary number of additional domains 685 within the system, we found that such domains turned out to be degenerate with the two 686 specified above. Therefore, we will not include these possibilities here. The effective enzyme ${ }_{687}$ profile is therefore of the form $e_{S} \delta(x)+e(x) \Theta\left(x_{0}-x\right)$, where $\delta(x)$ is the Dirac delta function 688 and $\Theta(x)$ is the Heaviside function.

The Lagrangian for this specific model is

$$
\begin{aligned}
\mathcal{L} & =\kappa e_{S} \rho^{(1)}(0)+\int_{0}^{x_{0}} \kappa e(x) \rho^{(1)}(x) \mathrm{d} x-\lambda_{e}\left(e_{S}+\int_{0}^{x_{0}} e(x) \mathrm{d} x-E_{T}\right) \\
& +\int_{0}^{x_{0}} \lambda^{(1)}(x)\left[D \partial_{x}^{2} \rho^{(1)}(x)-\kappa e(x) \rho^{(1)}(x)\right] \mathrm{d} x-\int_{x_{0}}^{L} \lambda^{(2)}(x) D \partial_{x}^{2} \rho^{(2)}(x) \mathrm{d} x \\
& +\lambda_{0}\left[j_{0}+D \partial_{x} \rho^{(1)}(0)-\kappa e_{S} \rho^{(1)}(0)\right]-\lambda_{L} \rho^{(2)}(L) \\
& +\lambda_{x_{0}}\left[\rho^{(1)}\left(x_{0}\right)-\rho^{(2)}\left(x_{0}\right)\right]+\lambda_{x_{0}}^{\prime}\left[\partial_{x} \rho^{(1)}\left(x_{0}\right)-\partial_{x} \rho^{(2)}\left(x_{0}\right)\right],
\end{aligned}
$$

690 where we have introduced the catalytic efficiency $\kappa=k_{\text {cat }} / K_{\mathrm{M}}$. Here $\lambda_{e}$ is the Lagrange mul691 tiplier corresponding to the constraint of having a fixed amount of enzymes $E_{T} ; \lambda^{(1)}(x)$ and ${ }_{692} \lambda^{(2)}(x)$ those corresponding the constrained dynamics of the substrate in the two domains; ${ }_{693} \lambda_{0}$ and $\lambda_{L}$ to the boundary conditions; and $\lambda_{x_{0}}$ and $\lambda_{x_{0}}^{\prime}$ to the continuity and smoothness 694 that must be imposed on $\rho(x)$ at the interface $x_{0}$.

To calculate the optimal enzyme arrangement $e_{S}^{*}, e^{*}(x)$ we must compute the various functional derivatives of $\mathcal{L}$. Let us first consider the derivatives with respect to $e(x)$ and $\rho^{(1)}(x)$. After integrating by parts Eq. B1 (corresponding to the form Eq. A4 of the Lagrangian), we find

$$
\begin{array}{ccc}
\frac{\delta \mathcal{L}}{\delta e(x)}=0 & \Leftrightarrow & \rho^{(1)}(x)=\frac{\lambda_{e}}{\kappa\left[1-\lambda^{(1)}(x)\right]} \\
\frac{\delta \mathcal{L}}{\delta \rho^{(1)}(x)}=0 & \Leftrightarrow & e(x)=-\frac{D \partial_{x}^{2} \lambda^{(1)}(x)}{\kappa\left[1-\lambda^{(1)}(x)\right]} .
\end{array}
$$


${ }_{695}$ We can compute the derivatives $\partial_{x} \rho^{(1)}(x)$ and $\partial_{x}^{2} \rho^{(1)}(x)$ starting from the expressions above 696 and we can substitute the results into the reaction-diffusion equation (given also by $\frac{\delta \mathcal{L}}{\delta \lambda^{(1)}(x)}=$ $6970)$ to obtain an ordinary differential equation for $\lambda^{(1)}(x)$ :

$$
\frac{\partial_{x}^{2} \lambda^{(1)}(x)}{\partial_{x} \lambda^{(1)}(x)}=-\frac{\partial_{x} \lambda^{(1)}(x)}{1-\lambda^{(1)}(x)} .
$$

${ }_{698}$ The solution to Eq. B4 can be found by direct integration to be

$$
\lambda^{(1)}(x)=1-A_{1} e^{B_{1} x}
$$

where $A_{1}$ and $B_{1}$ are constants of integration still to be determined. Using Eqs. B2-B3 we obtain

$$
\begin{aligned}
& \rho^{(1)}(x)=\frac{\lambda_{e}}{\kappa A_{1}} e^{-B_{1} x} \\
& e(x)=\frac{D B_{1}^{2}}{\kappa}
\end{aligned}
$$

${ }_{699}$ Equation B7 immediately shows that the enzyme density in the region $0 \leq x<x_{0}$ is 700 constant, in agreement with previous numerical studies [17, 18].

701 Evaluating the functional derivative of $\mathcal{L}$ with respect to $\lambda^{(2)}$, we recover the diffusion 702 equation $\partial_{x}^{2} \rho^{(2)}(x)=0$ in the domain $V_{2}$, from which

$$
\rho^{(2)}(x)=A_{2}+B_{2} x
$$

703 where $A_{2}$ and $B_{2}$ are again constants of integration.

704 The constants $A_{1,2}$ and $B_{1,2}$ can be evaluated using the boundary conditions at $x=0$ 705 and $x=L$, and the continuity and smoothness conditions at $x=x_{0}$. Additionally, the 706 dependence on $e_{S}$ can be eliminated using $\frac{\delta \mathcal{L}}{\delta \lambda_{e}}=0$, which is simply the constraint on the 707 total enzyme number. This leaves us with an expression for the Lagrangian that, since all 708 constraints have been satisfied equals the constrained reaction flux $J^{\mathcal{P}}$, in terms of the single 709 optimization variable $x_{0}$,

$$
\mathcal{L}=J^{\mathcal{P}}=j_{0}\left[1-\frac{1-\frac{x_{0}}{L}}{1-2 \frac{x_{0}}{L}+\alpha E_{T}\left(1-\frac{x_{0}}{L}\right)^{2}} e^{-\frac{x_{0}}{L-x_{0}}}\right]
$$

710 where we define $\alpha=\kappa L / D$. Finally, we maximize over $x_{0}$ to find

$$
x_{0}^{*}=\left\{\begin{array}{ll}
0 & \alpha E_{T} \leq 1 \\
L\left[1-\left(\alpha E_{T}\right)^{-1 / 2}\right] & \alpha E_{T}>1
\end{array} .\right.
$$

71 By substitution we can then obtain expressions for the enzyme and substrate densities 


\begin{tabular}{c|c|c|c|c|c} 
Regime & $e_{S}^{*}$ & $e^{*}(x)$ & $\rho^{(1)}(x)$ & $\rho^{(2)}(x)$ & $J^{\mathcal{P} *}$ \\
\hline \hline$\alpha E_{T} \leq 1$ & $E_{T}$ & 0 & $\mathrm{~N} / \mathrm{A}$ & $\frac{j_{0}(L-x)}{D\left(1+\alpha E_{T}\right)}$ & $\frac{j_{0}}{1+\left(\alpha E_{T}\right)^{-1}}$ \\
\hline \hline$\alpha E_{T}>1$ & $\sqrt{\frac{E_{T}}{\alpha}}$ & $\frac{E_{T}}{L}$ & $\frac{j_{0}}{2} \frac{L}{D \sqrt{\alpha E_{T}}} \exp \left[-\sqrt{\alpha E_{T}} \frac{x}{L}\right]$ & $\frac{j_{0}}{2} \frac{L-x}{D \sqrt{\alpha E_{T}}} \exp \left[-\sqrt{\alpha E_{T}} \frac{x_{0}}{L}\right]$ & $j_{0}\left(1-\frac{1}{2} e^{1-\sqrt{\alpha E_{T}}}\right)$
\end{tabular}

Table B.1. Optimal enzyme distribution and corresponding substrate profile and flux for a onedimensional system

\section{Appendix C: Marginal returns landscape}

714 Given a particular enzyme arrangement, the marginal returns as a function of position can 715 be calculated directly by considering the addition of an amount $\delta e$ of enzymes at position $x^{\prime}$, 716 and solving the new modified reaction-diffusion system. Here we present an example of such 717 a calculation. We consider the same one-dimensional system analyzed above, and suppose 718 that we are in the regime $\alpha E_{T}>1$ and the enzyme profile is the optimal one of Table B.1. ${ }_{719}$ For other enzyme profiles, such as those shown in Fig. $2 \mathrm{C}$ of the main text, the calculation 720 proceeds in the same way.

721 We now have to consider two cases: $0 \leq x^{\prime} \leq x_{0}$ and $x_{0}<x^{\prime} \leq L$.

If $0 \leq x^{\prime}<x_{0}$, we solve the reaction-diffusion equation in the three domains: $0 \leq x<x^{\prime}$, $x^{\prime}<x \leq x_{0}$, and $x_{0} \leq x \leq L$. The resulting density profiles are

$$
\begin{aligned}
& \rho_{\left[0, x^{\prime}\right]}(x)=a_{1} e^{\sqrt{\frac{E_{T^{\kappa}}}{D L}} x}+b_{1} e^{-\sqrt{\frac{E_{T^{\kappa}} \kappa}{D L}} x} \\
& \rho_{\left[x^{\prime}, x_{0}\right]}(x)=a_{2} e^{\sqrt{\frac{E_{T^{\kappa}}}{D L}} x}+b_{2} e^{-\sqrt{\frac{E_{T^{\kappa}}}{D L}} x} \\
& \rho_{\left[x_{0}, L\right]}(x)=a_{3} x+b_{3} .
\end{aligned}
$$

To fix the constants of integration $a_{i}, b_{i}$, we make use of the boundary conditions at $x=0$ and $x=L$ as well as matching conditions on $\rho$ at $x^{\prime}$ and $x_{0}$. Specifically, we require continuity of $\rho$ at $x^{\prime}$ and $x_{0}$, and smoothness at $x_{0}$,

$$
\begin{aligned}
\rho_{\left[0, x^{\prime}\right]}\left(x^{\prime}\right) & =\rho_{\left[x^{\prime}, x_{0}\right]}\left(x^{\prime}\right) \\
\rho_{\left[x^{\prime}, x_{0}\right]}\left(x_{0}\right) & =\rho_{\left[x_{0}, L\right]}\left(x_{0}\right) \\
\partial_{x} \rho_{\left[x^{\prime}, x_{0}\right]}\left(x_{0}\right) & =\partial_{x} \rho_{\left[x_{0}, L\right]}\left(x_{0}\right) .
\end{aligned}
$$

722 Additionally, conservation of mass at $x=x^{\prime}$ requires that

$$
D \partial_{x} \rho_{\left[x^{\prime}, x_{0}\right]}\left(x^{\prime}\right)-D \partial_{x} \rho_{\left[0, x^{\prime}\right]}\left(x^{\prime}\right)=\kappa \delta e \rho_{\left[x^{\prime}, x_{0}\right]}\left(x^{\prime}\right) .
$$


${ }_{723}$ The total flux $J^{\mathcal{P}}$ is then given by

$$
J^{\mathcal{P}}\left(x^{\prime}, \delta e\right)=\kappa\left[e_{S}^{*} \rho(0)+\delta e \rho\left(x^{\prime}\right)+\int_{0}^{x_{0}} e^{*}(x) \rho(x) \mathrm{d} x\right],
$$

724 into which we substitute the final density profile $\rho(x)$ as well as $e_{S}^{*}$ and $e^{*}(x)$ from Table B.1. ${ }_{725}$ Finally, the marginal returns at position $x^{\prime}$ can be calculated as

$$
\frac{d J^{\mathcal{P}}}{d e\left(x^{\prime}\right)}=\lim _{\delta e \rightarrow 0} \frac{\partial J^{\mathcal{P}}}{\partial \delta e}=\frac{j_{0}}{4} \sqrt{\frac{\alpha}{E_{T}}} e^{1-\sqrt{\alpha E_{T}}}
$$

726 Therefore, for $0 \leq x^{\prime} \leq x_{0}^{*}$ the marginal returns do not depend on $x^{\prime}$, and all positions at 727 which we have enzymes give the same returns. It is straightforward to verify that this agrees ${ }_{728}$ with the value of $\lambda_{e}$ derived from the optimal reaction flux that appears in Table B.1,

$$
\lambda_{e}=\frac{d J^{\mathcal{P} *}}{d E_{T}}=\frac{j_{0}}{4} \sqrt{\frac{\alpha}{E_{T}}} e^{1-\sqrt{\alpha E_{T}}}
$$

729 In the case $x_{0} \leq x^{\prime}<L$, the same procedure can be applied, except with the three 730 domains $0 \leq x \leq x_{0}, x_{0} \leq x \leq x^{\prime}$, and $x^{\prime} \leq x \leq L$. We find that the marginal returns is 731 given by

$$
\frac{d J^{\mathcal{P}}}{d e\left(x^{\prime}\right)}=\frac{j_{0} \alpha}{4} e^{1-\sqrt{\alpha E_{T}}}\left(1-\frac{x^{\prime}}{L}\right)\left(1+\sqrt{\alpha E_{T}} \frac{x^{\prime}-x_{0}}{L}\right) .
$$

732 As expected, this approaches Eq. C9 as $x^{\prime} \rightarrow x_{0}^{*}=L\left[1-\left(\alpha E_{T}\right)^{-1 / 2}\right]$.

\section{Appendix D: Derivation of the transition condition}

${ }_{734}$ In this section we derive the inequality condition, Eq. 10 of the main text, that determines 735 whether or not enzymes should be placed in the volume adjacent to a boundary position $\mathbf{s}$. ${ }_{736}$ We begin by examining the functional derivative of $\mathcal{L}$ with respect to substrate profiles at 737 the system boundary, $\rho(\mathbf{s})$. In the vicinity of a reflecting boundary, (i.e. $h(\mathbf{s})=0, k(\mathbf{s})=1)$, 738 we obtain from Eq. A4

$$
\frac{\delta \mathcal{L}}{\delta \rho(\mathbf{s})}=k_{\mathrm{cat}} e_{S}(\mathbf{s}) \frac{\delta F[\rho]}{\delta \rho}\left\{1-\lambda_{S}(\mathbf{s})\right\}-D \frac{\partial \lambda_{V}}{\partial \mathbf{n}(\mathbf{s})}-\left[\lambda_{V}(\mathbf{s})-\lambda_{S}(\mathbf{s})\right] \mathbf{v}(\mathbf{s}) \cdot \hat{\mathbf{n}}(\mathbf{s})=0
$$

739 where equality with zero assumes that the enzyme distribution is the optimal one. We can 740 also consider the functional derivative with respect to the gradient of $\rho(\mathbf{r})$ at the boundaries 741 of the system,

$$
\frac{\delta \mathcal{L}}{\delta \frac{\partial \rho}{\partial \mathbf{n}(\mathbf{s})}}=D\left[\lambda_{V}(\mathbf{s})-\lambda_{S}(\mathbf{s})\right]=0
$$


${ }_{742}$ Equation D2 requires $\lambda_{V}(\mathbf{s})=\lambda_{S}(\mathbf{s})$, which simplifies Eq. D1 to

$$
k_{\text {cat }} e_{S}(\mathbf{s}) F^{\prime}[\rho(\mathbf{s})]\left\{1-\lambda_{V}(\mathbf{s})\right\}-D \frac{\partial \lambda_{V}}{\partial \mathbf{n}(\mathbf{s})}=0,
$$

743 where $F^{\prime}[\rho(\mathbf{s})]=\frac{\delta F[\rho]}{\delta \rho}$.

We next examine variations of $\mathcal{L}$ with respect to the enzyme densities,

$$
\begin{gathered}
\frac{\delta \mathcal{L}}{\delta e_{S}(\mathbf{s})}=k_{\mathrm{cat}} F[\rho(\mathbf{s})]\left\{1-\lambda_{S}(\mathbf{s})\right\}-\lambda_{e} \leq 0 \\
\frac{\delta \mathcal{L}}{\delta e(\mathbf{r})}=k_{\mathrm{cat}} F[\rho(\mathbf{r})]\left\{1-\lambda_{V}(\mathbf{r})\right\}-\lambda_{e} \leq 0 .
\end{gathered}
$$

${ }_{744}$ Both $\lambda_{V}(\mathbf{r})$ and $\rho(\mathbf{r})$ must be continuous, since they must both satisfy (generally non-linear) 745 diffusion equations. Therefore, it follows from Eq. D5 that $\frac{\delta \mathcal{L}}{\delta e(\mathbf{r})}$ is itself continuous and that 746 we can define the spatial gradient $\nabla \frac{\delta \mathcal{L}}{\delta e(\mathbf{r})}$. At all points where it is optimal to allocate a non747 zero density of enzymes (both in the interior of the system or on the boundaries) we have ${ }_{748}$ that $\frac{\delta \mathcal{L}}{\delta e_{S}(\mathbf{s})}=\frac{\delta \mathcal{L}}{\delta e(\mathbf{r})}=0$, implying that $\nabla \frac{\delta \mathcal{L}}{\delta e(\mathbf{r})}=0$. In regions where enzymes are not present 749 because it is suboptimal to add them, we get that $\frac{\delta \mathcal{L}}{\delta e(\mathbf{r})}<0$, and the gradient $\nabla \frac{\delta \mathcal{L}}{\delta e(\mathbf{r})}$ will in 750 general be finite. Furthermore, since $\rho(\mathbf{r}) \rightarrow \rho(\mathbf{s})$ and $\lambda_{V}(\mathbf{r}) \rightarrow \lambda_{V}(\mathbf{s})=\lambda_{S}(\mathbf{s})$ smoothly as ${ }_{751} \mathbf{r} \rightarrow \mathbf{S}, \nabla \frac{\delta \mathcal{L}}{\delta e(\mathbf{r})}$ must remain well defined as we approach the system boundaries.

752 If it is optimal to have enzymes at the boundaries of the system but not locally in the 753 interior, $\frac{\delta \mathcal{L}}{\delta e_{S}(\mathbf{s})}=0$ and $\frac{\delta \mathcal{L}}{\delta e(\mathbf{r})}<0$ for a small displacement $\mathbf{r}=\mathbf{s}+\boldsymbol{\epsilon}$. In this case, evaluating 754 the gradient of Eq. D5 and taking $\mathbf{r} \rightarrow \mathbf{s}$ we find

$$
\frac{\partial}{\partial \mathbf{n}(\mathbf{s})} \frac{\delta \mathcal{L}}{\delta e(\mathbf{r})}=k_{\text {cat }}\left[F^{\prime}[\rho(\mathbf{s})]\left(1-\lambda_{V}(\mathbf{s})\right) \frac{\partial \rho}{\partial \mathbf{n}(\mathbf{s})}-F[\rho(\mathbf{s})] \frac{\partial \lambda_{V}}{\partial \mathbf{n}(\mathbf{s})}\right]>0 .
$$

755 The inequality Eq. D6, $\frac{\partial}{\partial \mathbf{n}(\mathbf{s})} \frac{\delta \mathcal{L}}{\delta e(\mathbf{r})}>0$, is equivalent to Eq. 9 of the main text, $\frac{\partial}{\partial \mathbf{n}(\mathbf{s})} \frac{d J^{\mathcal{P}}}{d e}>0$, 756 because $\frac{\delta \mathcal{L}}{\delta e(\mathbf{r})}=\frac{d J^{\mathcal{P}}}{d e(\mathbf{r})}-\lambda_{e}$ and $\lambda_{e}$ is constant. Hence $\frac{\partial}{\partial \mathbf{n}(\mathbf{s})} \frac{\delta \mathcal{L}}{\delta e(\mathbf{r})}=\frac{\partial}{\partial \mathbf{n}(\mathbf{s})} \frac{d J^{\mathcal{P}}}{d e}$. Substituting for $757 \frac{\partial \lambda_{V}}{\partial \mathbf{n}(\mathbf{s})}$ from Eq. D3, this inequality becomes

$$
k_{\text {cat }} F^{\prime}[\rho(\mathbf{s})]\left(1-\lambda_{V}(\mathbf{s})\right)\left\{\frac{\partial \rho}{\partial \mathbf{n}(\mathbf{s})}-\frac{k_{\text {cat }}}{D} e_{S}(\mathbf{s}) F[\rho(\mathbf{s})]\right\}>0 .
$$

758 Since we assume that $F[\rho]$ is monotonically increasing, $F^{\prime}[\rho]>0$. Furthermore, setting 759 Eq. D4 equal to zero implies that $1-\lambda_{V}(\mathbf{s})>0$, since $\lambda_{e}>0$. Therefore, Eq. D7 reduces to

$$
D \frac{\partial \rho}{\partial \mathbf{n}(\mathbf{s})}>k_{\text {cat }} e_{S}(\mathbf{s}) F[\rho(\mathbf{s})]
$$

760 which corresponds to Eq. 10 of the main text. 


\section{Appendix E: Reduction of numerical complexity in a one-dimensional system with} 762 drift

In this section we demonstrate how, even in cases for which the full analytical solution 764 is not tractable, it is nevertheless possible to use the Lagrangian formalism to express the 765 optimization problem as a single equation, which can then be solved numerically.

766 We consider a one-dimensional system, similar to that considered in Section B but with 767 the addition of a constant drift $v$. If $v>0$ substrate molecules tend to flow away from the 768 source at the origin, whereas for $v<0$ they tend to flow towards it. The corresponding 769 Lagrangian is

$$
\begin{aligned}
\mathcal{L} & =\kappa e_{S} \rho^{(1)}(0)+\int_{0}^{x_{0}} \kappa e(x) \rho^{(1)}(x) \mathrm{d} x-\lambda_{e}\left(e_{S}+\int_{0}^{x_{0}} e(x) \mathrm{d} x-E_{T}\right) \\
& +\int_{0}^{x_{0}} \lambda^{(1)}(x)\left[D \partial_{x}^{2} \rho^{(1)}(x)-v \partial_{x} \rho^{(1)}(x)-\kappa e(x) \rho^{(1)}(x)\right] \mathrm{d} x \\
& -\int_{x_{0}}^{L} \lambda^{(2)}(x)\left[D \partial_{x}^{2} \rho^{(2)}(x)-v \partial_{x} \rho^{(2)}(x)\right] \mathrm{d} x \\
& +\lambda_{0}\left[j_{0}+D \partial_{x} \rho^{(1)}(0)-v \rho^{(1)}(0)-\kappa e_{S} \rho^{(1)}(0)\right]-\lambda_{L} \rho^{(2)}(L) \\
& +\lambda_{x_{0}}\left[\rho^{(1)}\left(x_{0}\right)-\rho^{(2)}\left(x_{0}\right)\right]+\lambda_{x_{0}}^{\prime}\left[\partial_{x} \rho^{(1)}\left(x_{0}\right)-\partial_{x} \rho^{(2)}\left(x_{0}\right)\right] .
\end{aligned}
$$

The derivation proceeds in the same way as in Section B. We again use the functional derivatives to find expressions for $e(x)$ and $\rho^{(1)}(x)$ in terms of the Lagrange multiplier $\lambda^{(1)}(x)$,

$$
\begin{array}{cll}
\frac{\delta \mathcal{L}}{\delta e(x)}=0 \quad & \Leftrightarrow \quad \rho^{(1)}(x)=\frac{\lambda_{e}}{\kappa\left[1-\lambda^{(1)}(x)\right]} \\
\frac{\delta \mathcal{L}}{\delta \rho^{(1)}(x)}=0 \quad \Leftrightarrow \quad e(x)=-\frac{D \partial_{x}^{2} \lambda^{(1)}(x)+v \partial_{x} \lambda^{(1)}(x)}{\kappa\left[1-\lambda^{(1)}(x)\right]} .
\end{array}
$$

Surprisingly, when we combine these expressions with the reaction-diffusion equation with drift, we find that the resulting differential equation for $\lambda^{(1)}(x)$ is again exactly Eq. B4, which we can solve to find

$$
\begin{aligned}
& \lambda^{(1)}(x)=1-\tilde{A}_{1} e^{\tilde{B}_{1} x} \\
& \rho^{(1)}(x)=\frac{\lambda_{e}}{\kappa \tilde{A}_{1}} e^{-\tilde{B}_{1} x} \\
& e(x)=\frac{D \tilde{B}_{1}^{2}+v \tilde{B}_{1}}{\kappa} .
\end{aligned}
$$

770 Notably the optimal enzyme density $e(x)$ is again constant, but now depends on the drift 771 velocity $v$. 
772 In the region with no enzymes, $\rho^{(2)}(x)$ obeys a diffusion equation with drift, $D \partial_{x}^{2} \rho^{(2)}(x)-$ $773 v \partial_{x} \rho^{(2)}(x)=0$, which has the solution

$$
\rho^{(2)}(x)=\tilde{A}_{2}+\tilde{B}_{2} e^{\operatorname{Pe} x / L}
$$

774 where $\mathrm{Pe}=v L / D$ is the Péclet number. As before, we evaluate the integration constants

${ }_{775} \tilde{A}_{1,2}, \tilde{B}_{1,2}$ using the boundary conditions at $x=0$ and $x=L$, and the continuity and 776 smoothness conditions at $x_{0}$. We again eliminate the dependence on $e_{S}$ by using the con777 straint on the total enzyme number. We are left with an expression for $J^{\mathcal{P}}$ in terms of the 778 single optimization variable $x_{0}$,

$$
\mathcal{L}=J^{\mathcal{P}}=j_{0}\left\{1-\frac{\exp \left\{-\operatorname{Pe} \frac{x_{0}}{L}\left[e^{\operatorname{Pe}\left(1-x_{0} / L\right)}-1\right]^{-1}\right\}}{1+\frac{\alpha E_{T}}{\operatorname{Pe}}\left[1-e^{-\operatorname{Pe}\left(1-x_{0} / L\right)}\right]-\operatorname{Pe} \frac{x_{0}}{L}\left[e^{\operatorname{Pe}\left(1-x_{0} / L\right)}-1\right]^{-1}}\right\}
$$

779 where $\alpha$ is defined as in Section B. It can be shown that Eq. E8 reduces to Eq. B9 in the 780 limit $\mathrm{Pe} \rightarrow 0$.

781 It is not possible to find the $x_{0}^{*}$ that maximizes $J^{\mathcal{P}}$ analytically. However, it is straight782 forward to solve $\frac{d J^{\mathcal{P}}}{d x_{0}}=0$ numerically for specified values of the other parameters appearing 783 in Eq. E8. The results for the rescaled reaction flux and the rescaled net diffusive flux at 784 the origin are shown in Fig. 3 of the main text.

\section{Appendix F: One-dimensional system with two sources of substrate}

${ }_{786}$ Here we present an example of a scenario of multiple sources of substrate with different 787 strengths. We considered a one-dimensional system in the domain $0 \leq x \leq L$, with reflecting 788 boundaries at both ends. At $x=0$ we apply a source of substrate with $j(0)=1$, and at ${ }_{789} x=L$ we apply a source with $j(L)=0.3$. Instead of at the boundary, loss of substrate 790 occurs via decay with rate $\sigma$. We again assume linear reactions, $F[\rho]=\rho / K_{\mathrm{M}}$. For this 791 system we have identified a number of different regimes of the optimal enzyme arrangement 792 as a function of $E_{T}$, shown in Fig. F.1A.

793 At the lowest $E_{T}$ values, the optimal arrangement consists of a single cluster at $x=0$, 794 the site of strongest substrate influx. As $E_{T}$ crosses a first transition level, $E_{T}^{t 1}$, the optimal 795 configuration features clusters of enzymes at both ends of the system: a larger one at $x=0$ 796 (the stronger source) and a smaller one at $x=L$ (the weaker source). In this regime 797 the optimal distribution of enzymes between the two poles can be calculated exactly by 
A

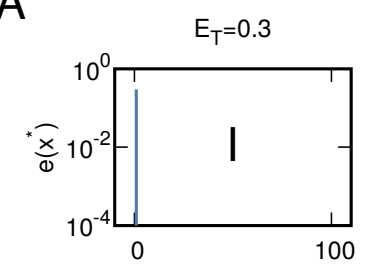

$\mathrm{E}_{\mathrm{T}}=0.6$

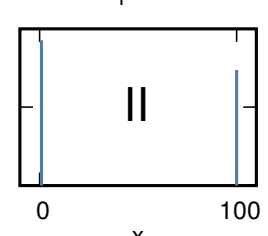

B

\begin{tabular}{|c|c|c|c|c|c|}
\hline $\mathrm{j}^{P} / \mathrm{j}$ & $\mathrm{j}^{\mathrm{D} / \mathrm{j}}$ & $\begin{array}{c}\text { Geometry } \\
\text { Symmetry }\end{array}$ & Reaction & $\sigma$ & $\mathrm{V}$ \\
\hline$-\Delta$ & $\cdots \cdots-\Delta$ & 1D two sources & $\begin{array}{l}\text { Linear } \\
\text { Linear }\end{array}$ & $\mathrm{Y}$ & $\mathrm{Y}$ \\
\hline & $\mathrm{N}$ \\
\hline
\end{tabular}

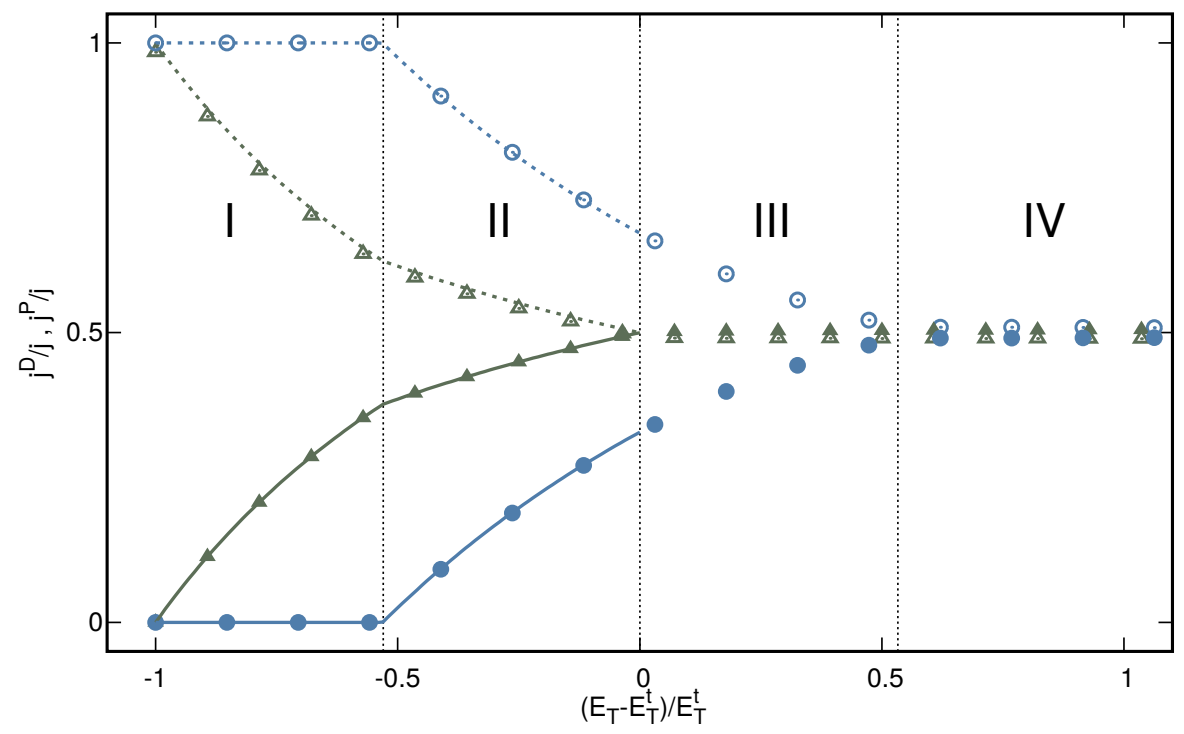

Figure F.1. Transitions in the optimal enzyme arrangement for a one-dimensional system with two sources of substrate. The system is defined by $\sigma=1, \alpha=L k_{\text {cat }} /\left(K_{\mathrm{M}} D\right)=1, v=0$ and boundary conditions $j(0)=1, k(0)=-1, h(0)=0$ and $j(L)=0.3, k(L)=-1, h(L)=0$. (A) Examples of the optimal enzyme profiles for different values of $E_{T}$. (B) Diffusion and reaction fluxes at $x=0$ and $x=L$, normalized by the local influxes of substrate, are plotted as a function of $\left(E_{T}-E_{T}^{t 2}\right) / E_{T}^{t 2}$. For both poles of the system, we confirm that $j^{D}>j^{\mathcal{P}}$ when the optimal enzyme distribution consists of only a single boundary cluster at that pole, while $j^{D}=j^{\mathcal{P}}=j / 2$ when the optimal enzyme arrangement extends a finite distance away from the pole. Lines show analytical solutions. Points show results of numerical optimization. Fig. 3 of the main text shows the same data with the fluxes at $x=L$ plotted against $\left(E_{T}-E_{T}^{t 3}\right) / E_{T}^{t 3}$. 
798 solving the reaction-diffusion equation with arbitrary cluster densities $e_{0}$ and $e_{L}$, and then 799 optimizing over $e_{0}$ and $e_{L}$ subject to the constraint $e_{0}+e_{L}=E_{T}$. The transition position $800 E_{T}^{t 1}$ is then the value at which the resulting optimal $e_{L}^{*}=0$. However, since the expressions 801 are large and complex we do not include them here.

${ }_{802}$ As $E_{T}$ is increased further we reach a second transition at $E_{T}^{t 2}$, with enzymes being 803 placed within the system in the vicinity of $x=0$. This transition occurs when the diffusive 804 and reaction fluxes at $x=0$ become equal, $j^{D}(0)=j^{\mathcal{P}}(0)=j(0) / 2$ (see Fig. F.1B). At 805 the transition value $E_{T}^{t 2}$, the corresponding fluxes at $x=L$ are not equal and still satisfy 806 the inequality condition Eq. D8 (Eq. 10 of the main text). Therefore, around $x=L$ the 807 optimal configuration is still purely clustered, with no enzymes at $x<L$. The transition 808 threshold $E_{T}^{t 2}$ can be determined exactly by calculating the fluxes $j^{D}(0)$ and $j^{\mathcal{P}}(0)$ from the 809 full solution of the system with the two optimal polar clusters.

810 At a larger transition value $E_{T}^{t 3}$, the diffusive and reactive fluxes at $x=L$ also become ${ }_{811}$ equal, $j^{D}(L)=j^{\mathcal{P}}(L)=j(L) / 2$ (see Fig. F.1B). For $E_{T}>E_{T}^{t 3}$, enzymes are present away 812 from the boundaries at both ends of the system. In the regime $E_{T}>E_{T}^{t 2}$ it is not possible ${ }_{813}$ to solve for the form of the optimal profile $e^{*}(x)$, or therefore to calculate $j^{D}(L)$ and $j^{\mathcal{P}}(L)$. ${ }_{814}$ The value of $E_{T}^{t 3}$ must therefore be determined numerically.

815 Finally, if $E_{T}$ is increased further, the enzyme density profiles extending from the two 816 ends of the system will eventually coalesce, and enzymes will be present at all positions ${ }_{817} 0 \leq x \leq L$

${ }_{818}$ It is also possible for these transitions to occur in a different order for different parameter 819 combinations. For example, if $j(L) \ll j(0)$, the order of the first two transitions may be 820 reversed. In this case, the condition $j^{D}(0)=j^{\mathcal{P}}(0)=j(0) / 2$ is satisfied before the transition 821 value $E_{T}^{t 1}$ is reached. The first transition will then be from a single cluster at $x=0$ to a 822 regime in which there is a finite extension of enzymes from $x=0$, but no cluster at $x=L$. ${ }_{823}$ The appearance of the second cluster at $x=L$ may then occur subsequently, at larger $E_{T}$, 824 resulting in an optimal enzyme profile than resembles type III in Fig. F.1A. 


\section{Appendix G: Optimal enzyme allocation algorithm for discretized systems}

\section{Derivation of the enzyme repartition rule}

827 In this section we derive Eqs. 12, 14 of the main text, which lead to the optimal enzyme 828 repartition rule used in step 5 of the construction algorithm. For the implementation of 829 an optimization algorithm, we consider a system with discretized space. In this model, 830 the densities of substrate and enzyme change from functions of position to a discrete set 831 of variables, $\left\{e_{i}\right\}=\left\{e\left(\mathbf{r}_{i}\right)\right\}$ for $i=1 . . N$, where $N$ is the number of lattice sites. Instead 832 of being a functional of $e(\mathbf{r}), \rho(\mathbf{r})$, the corresponding discrete Lagrangian then becomes a 833 function of $\left\{e_{i}\right\},\left\{\rho_{i}\right\}$, and functional derivatives $\frac{\delta \mathcal{L}}{\delta f(\mathbf{r})}$ become normal derivatives $\frac{\partial \mathcal{L}}{\partial f_{i}}$.

${ }_{834}$ The derivative of $\mathcal{L}$ with respect to the enzyme density has an analogous form as in the 835 continuous system,

$$
\frac{\partial \mathcal{L}}{\partial e_{i}}=k_{\mathrm{cat}} F\left[\rho_{i}\right]\left\{1-\lambda_{i}\right\}-\lambda_{e}
$$

836 where $\lambda_{i}$ is the Lagrange multiplier associated with the discrete reaction-diffusion equation 837 at site $i$. We again denote the first term above by $\frac{d J^{\mathcal{P}}}{d e_{i}}$, with the same interpretation as in the 838 continuous case. This quantity is generally easily accessible in any numerical analysis: one 839 simply increases the amount of enzymes at position $i$ by a small amount, solves the resulting 840 modified reaction-diffusion system, and calculates the resulting change in the total reaction ${ }_{841}$ flux. In the same way as for the continuous system, for the optimal enzyme arrangement ${ }_{842}\left\{e_{i}^{*}\right\}$ we must have that $\frac{\partial \mathcal{L}}{\partial e_{i}}=0$ at all sites $i$ where $e_{i}^{*}>0$, and $\frac{\partial \mathcal{L}}{\partial e_{i}} \leq 0$ where $e_{i}^{*}=0$.

843 We now consider the optimal amount of enzymes at site $i$ to be a function of $E_{T}, e_{i}^{*}\left(E_{T}\right)$. 844 That is, there is a line $\mathbf{e}^{*}\left(E_{T}\right)$ in the space of $\mathbf{e}=\left(e_{1}, e_{2}, \ldots, e_{N}\right)$, that we can parameterize 845 by the value of $E_{T}$, for which the reaction flux $J_{\mathcal{P}}\left(\mathbf{e}^{*}\left(E_{T}\right)\right)$ is the highest that can be achieved 846 for that value of $E_{T}$. We begin by considering the derivative of $\frac{d J^{\mathcal{P}}}{d e_{i}}$ with respect to $E_{T}$ along 847 the line defined by $\mathbf{e}^{*}\left(E_{T}\right), \frac{d}{d E_{T}} \frac{d J^{\mathcal{P}}}{d e_{i}}$. We now expand the total derivative $\frac{d}{d E_{T}}$ in terms of ${ }_{848}$ derivatives with respect to the underlying variables $\left\{e_{i}\right\}$,

$$
\frac{d}{d E_{T}} \frac{d J^{\mathcal{P}}}{d e_{i}}=\sum_{j=1}^{N} \frac{d e_{j}}{d E_{T}} \frac{d}{d e_{j}} \frac{d J^{\mathcal{P}}}{d e_{i}}
$$

849 Note that here $\frac{d}{d e}$ takes into account the changes in the flux both due directly to altering 850 the enzyme density, and the resulting change in the substrate density profile $\left\{\rho_{i}\right\}$. Since ${ }_{851} \frac{d e_{j}}{d E_{T}}$ is understood to be in the direction of the line of optimal profiles, it coincides with $\frac{d e_{j}^{*}}{d E_{T}}$. 
${ }_{852}$ Writing the set of $N$ equations Eq. G2 in vector form we have

$$
\frac{d}{d E_{T}} \frac{d J^{\mathcal{P}}}{d \mathbf{e}}=\mathbf{H} \frac{d \mathbf{e}^{*}}{d E_{T}},
$$

853 where $\mathbf{H}$ is the Hessian of $J^{\mathcal{P}}, H_{i j}=\frac{d^{2} J^{\mathcal{P}}}{d e_{j} d e_{i}}$.

${ }_{854}$ From Eq. G1 we know that if there are $1 \leq n \leq N$ positions at which the optimal enzyme 855 density is non-zero, then

$$
\left(\begin{array}{c}
\frac{d J^{\mathcal{P}}}{d e_{1}} \\
\vdots \\
\frac{d J^{\mathcal{P}}}{d e_{n}} \\
\frac{d J^{\mathcal{P}}}{d e_{n+1}} \\
\vdots \\
\frac{d J^{\mathcal{P}}}{d e_{N}}
\end{array}\right)=\lambda_{e}\left(\begin{array}{c}
1 \\
\vdots \\
1 \\
c_{n+1} \\
\vdots \\
c_{N}
\end{array}\right)
$$

${ }_{856}$ where all $c^{\prime}$ s lie in the range $0<c_{i}<1$, and we have numbered lattice sites in descending 857 order of marginal returns. Therefore, Eq. G3 becomes

$$
\mathbf{H} \frac{d \mathbf{e}^{*}}{d E_{T}}=\frac{d \lambda_{e}}{d E_{T}}\left(\begin{array}{c}
1 \\
\vdots \\
1 \\
c_{n+1}+\frac{d c_{n+1}}{d E_{T}} \frac{\lambda_{e}}{\frac{d \lambda_{e}}{d E_{T}}} \\
\vdots \\
c_{N}+\frac{d c_{N}}{d E_{T}} \frac{\lambda_{e}}{\frac{d \lambda_{e}}{d E_{T}}}
\end{array}\right) .
$$

${ }_{858}$ In general it is not possible to directly compute the right hand side of Eq. G5, because 859 without knowing the direction of the optimal line it is not possible to calculate the derivatives $860 \frac{d \lambda_{e}}{d E_{T}}$ and $\frac{d c_{i}}{d E_{T}}$. However, exploiting the fact that no enzymes should be added to the system at ${ }_{861}$ positions where $\frac{d J^{\mathcal{P}}}{d e_{i}}<\lambda_{e}$, the vector $\frac{d \mathbf{e}^{*}}{d E_{T}}$ must have zeros at all positions $i>n$. Therefore, 862 the $N$-dimensional system in Eq. G5 can be split into two parts. Primarily, we have the $863 n$-dimensional system

$$
\mathbf{H}_{(n)} \frac{d \mathbf{e}_{(n)}^{*}}{d E_{T}}=\frac{d \lambda_{e}}{d E_{T}} \mathbf{1}_{(n)},
$$

${ }_{864}$ where the subscript $(n)$ denotes that only the first $n$ positions of the lattice are considered. ${ }_{865}$ Importantly, in order to solve Eq. G6 it is not necessary to know $\frac{d \lambda_{e}}{d E_{T}}$ in advance. Instead, one ${ }_{866}$ can solve for any constant prefactor, and then subsequently use the normalization condition 
${ }_{867} \sum_{i=1}^{n} \frac{d e_{i}^{*}}{d E_{T}}=1$ to express $\frac{d \lambda_{e}}{d E_{T}}$ in terms of this constant. For self-consistency, the solution to ${ }_{868}$ Eq. G6 must also solve the residual $(N-n)$-dimensional system

$$
\left(\begin{array}{cccc}
\frac{d^{2} J^{\mathcal{P}}}{d e_{1} d e_{n+1}} & \frac{d^{2} J^{\mathcal{P}}}{d e_{2} d e_{n+1}} & \cdots & \frac{d^{2} J^{\mathcal{P}}}{d e_{n} d e_{n+1}} \\
\vdots & \vdots & \ddots & \vdots \\
\frac{d^{2} J^{\mathcal{P}}}{d e_{1} d e_{N}} & \frac{d^{2} J^{\mathcal{P}}}{d e_{2} d e_{N}} & \cdots & \frac{d^{2} J^{\mathcal{P}}}{d e_{n} d e_{N}}
\end{array}\right) \frac{d \mathbf{e}_{n}^{*}}{d E_{T}}=\frac{d \lambda_{e}}{d E_{T}}\left(\begin{array}{c}
c_{n+1}+\frac{d c_{n+1}}{d E_{T}} \frac{\lambda_{e}}{\frac{d \lambda_{e}}{d E_{T}}} \\
\vdots \\
c_{N}+\frac{d c_{N}}{d E_{T}} \frac{\lambda_{e}}{\frac{d \lambda_{e}}{d E_{T}}}
\end{array}\right)
$$

869 which can be interpreted as defining the evolution of the marginal returns at positions 870 without enzymes, $\frac{d c_{i}}{d E_{T}}$.

\section{Discretization effects}

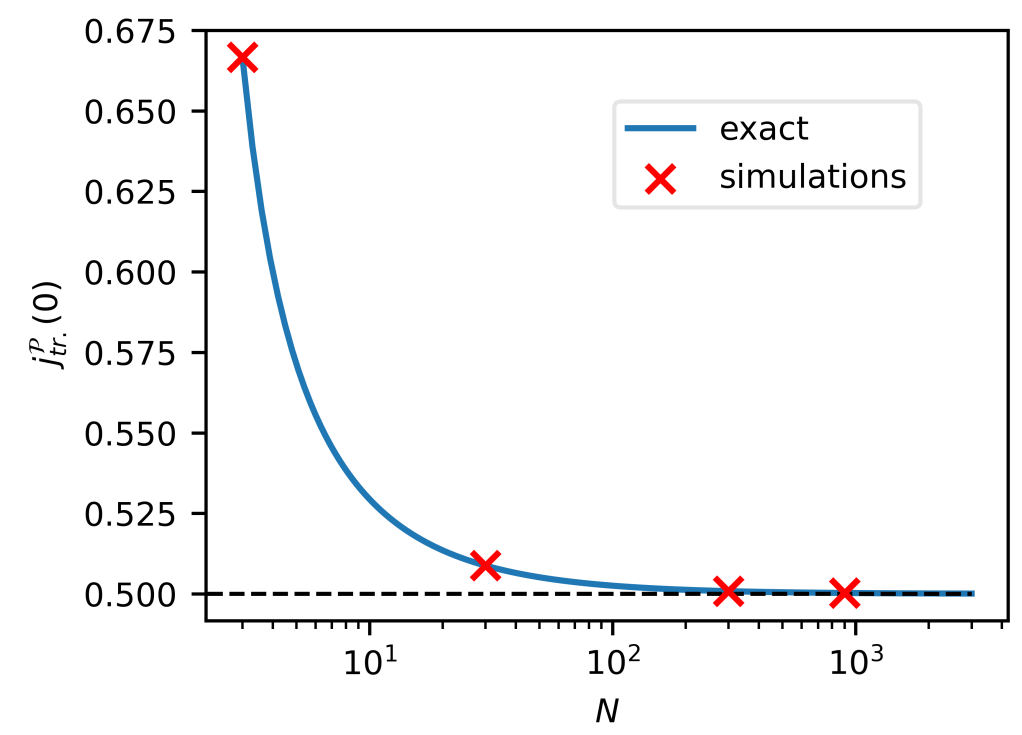

Figure G.1. Value of the reaction flux at the transition $j^{\mathcal{P}}(\mathbf{s})$ vs the number of lattice sites $N$. The blue line is the exact solution obtained by iteratively solving the discrete reaction-diffusion system at steady-state, while the red crosses are the result of the construction algorithm for different $N$ values.

872 How large should the number of lattice sites $N$ be to have a good approximation of the 873 continuum limit of the system? In this section we discuss a criterion to find such a value.

874 In the main text we derive a general condition for the transition in the optimal enzyme 875 arrangement from a localized arrangement into an extended profile. The condition (Eq. 10 
876 of the main text), in combination with the boundary condition, at the transition, reads:

$$
j^{\mathcal{P}}(\mathbf{s})=\frac{1}{2}(j(\mathbf{s})-\rho(\mathbf{s}) \mathbf{v}(\mathbf{s}) \cdot \hat{n}(\mathbf{s}))
$$

877 which becomes $j^{\mathcal{P}}(\mathbf{s})=j(\mathbf{s}) / 2$ in case of no advection $\mathbf{v}=0$. This condition does not 878 depend on the geometry, nor on the loss mechanism considered for the substrate and it 879 applies locally at every position of the system boundaries with influx of substrate. As can 880 be seen in Appendix D, the above condition is equivalent to $\frac{\partial}{\partial \mathbf{n}(\mathbf{s})} \frac{d J^{\mathcal{P}}}{d e}=0$ and in general is 881 derived by combining different derivatives of the Lagrangian $\mathcal{L}$. The derivatives are going 882 to be affected by the system discretization, implying that Eq. G8 is valid only in the limit ${ }_{883} N \rightarrow \infty$.

${ }_{884}$ For example in the case of the discrete version of the system described in Fig. 2 of the 885 main text and solved in Appendix B, C, it can be shown by solving iteratively the reaction886 diffusion equation, that the transition happens for $\alpha E_{T}^{t}=N /(N-2)$ and that the reaction ${ }_{887}$ flux at the transition is $j^{\mathcal{P}}(0)=j(0)(N-1) /(2 N-3)$. In the limit of $N \rightarrow \infty$ the two 888 limits are $\alpha E_{T}^{t} \rightarrow 1$ and $j^{\mathcal{P}}(0) \rightarrow j(0) / 2$, which coincide with the continuum solution, cf. ${ }_{889}$ Appendix B. In Fig. G.1 we can see how $j^{\mathcal{P}}(0)$ scales as a function of the number of lattice 890 sites $N$. In blue is the exact solution, while the red crosses are the result of the construction 891 algorithm for different discretizations. For example, we can see how for $N=100$ the 892 discrepancy between the continuum limit and the discrete system is approximately $0.51 \%$.

\section{Efficiency of the construction algorithm}

${ }_{894}$ In this section we compare the computational times of the construction algorithm derived 895 in this work and a stochastic optimization algorithm that has been previously used to find the 896 optimal enzyme arrangements in various systems belonging to the class of systems illustrated 897 in Fig. 1 of the main text [17, 18].

${ }_{898}$ The stochastic optimization algorithm takes an initial uniform enzyme configuration. 899 Then, an iteration of the algorithm consists in taking a random amount of enzymes and 900 moving it from a random site to a different random site. This new enzyme configuration is 901 then the initial configuration for the next optimization step if the reaction flux generated 902 by the new enzyme profile is higher than the previous configuration. The optimization 903 is stopped after a maximum number of iterations of $N_{i t e r}=10^{5}$, or if the flux does not 
904 increase for $N_{i t e r}=10^{4}$. For each change in the enzyme arrangement, the steady-state 905 reaction-diffusion equation needs to be solved. The general form of the equation for a 906 discrete system in matrix form is (cf. Material and Methods of the main text):

$$
(\mathbf{D}+\mathbf{V}) \boldsymbol{\rho}-\alpha \mathbf{e} \odot \mathbf{F}-\sigma \boldsymbol{\rho}=\mathbf{A}
$$

${ }_{907}$ where $\mathbf{D}$ and $\mathbf{V}$ are the diffusion and advection operator on the lattice respectively, $\boldsymbol{\rho}$ the 908 substrate density, $\mathbf{F}$ is the vector with $F_{i}=F\left[\rho_{i}\right]$ and $\mathbf{A}$ the substrate influx vector, while $909 \odot$ denotes the element-wise (Hadamard) product.

${ }_{910}$ For the comparison of the two algorithms, we decided to consider a 1D system with two 911 sources of substrate and linear reactions as described in Appendix F. We consider such a 912 system because we expect the stochastic optimization algorithm to perform at its best in 1D 913 systems with linear reactions. For such systems the diffusion matrix $\mathbf{D}$ becomes a tridiagonal 914 symmetric matrix and Eq. G9 becomes a linear algebraic system that can be solved with 915 the use of a tridiagonal inversion algorithm that is $o(N)$. Since the random selection of sites 916 is $o\left(N^{2}\right)$, we expect the stochastic optimization algorithm with a tridiagonal solver to be $917 O\left(N^{3}\right)$.

${ }_{918}$ Our construction algorithm is also $o\left(N^{3}\right)$ since the Hessian inversion required in step 5 919 of the algorithm follows a Cholesky decomposition. Moreover, given the iterative nature of 920 our construction algorithm, we have a prefactor that increases with the target amount of 921 enzymes $E_{T}$ that we want to reach.

922 In Fig. G.2A we plot in blue circles the average computational time spent by the stochastic 923 optimization algorithm with a solver for tridiagonal symmetric matrices over 20 repeats and 924 with the green pluses we mark the computational time of the construction algorithm. The 925 standard deviation of the time spent by the stochastic optimization algorithm is not shown 926 as it is smaller than the size of the markers used. We can see that the construction algorithm 927 is faster than the stochastic optimization with tridiagonal solver only for low amounts of ${ }_{928} E_{T}$. Passed the first transition value $E_{T}^{t}$, the construction algorithm gets up to 10 times 929 slower than the stochastic optimization one with tridiagonal solver. Note, however, that the 930 stochastic optimization algorithm returns the optimal enzyme configuration for a single $E_{T}$ 931 value, whereas the construction algorithm gives the whole optimal trajectory up to the target 932 value $E_{T}$. Moreover, this is the limiting case of a system having a symmetric tridiagonal 933 diffusion operator. In general this is not the case, e.g. higher dimensional systems. In 
A

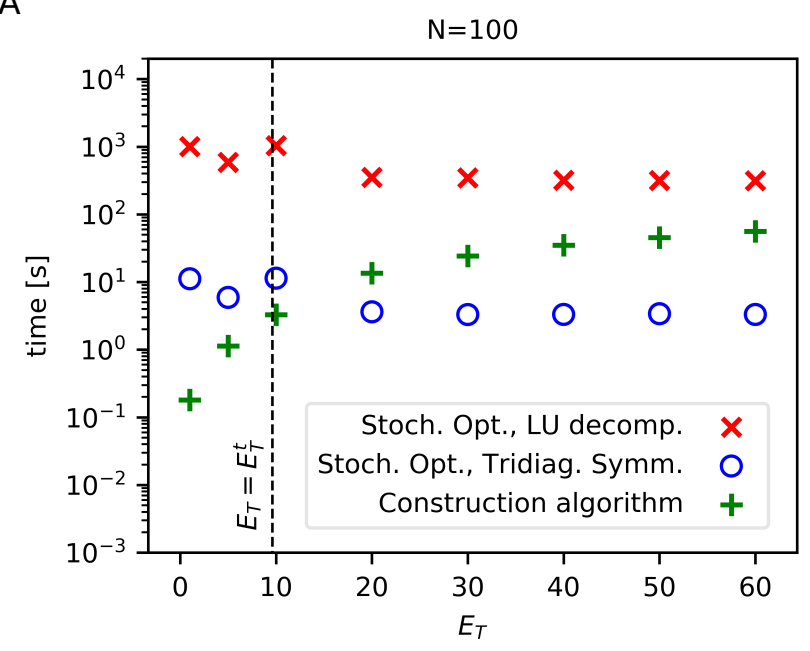

B

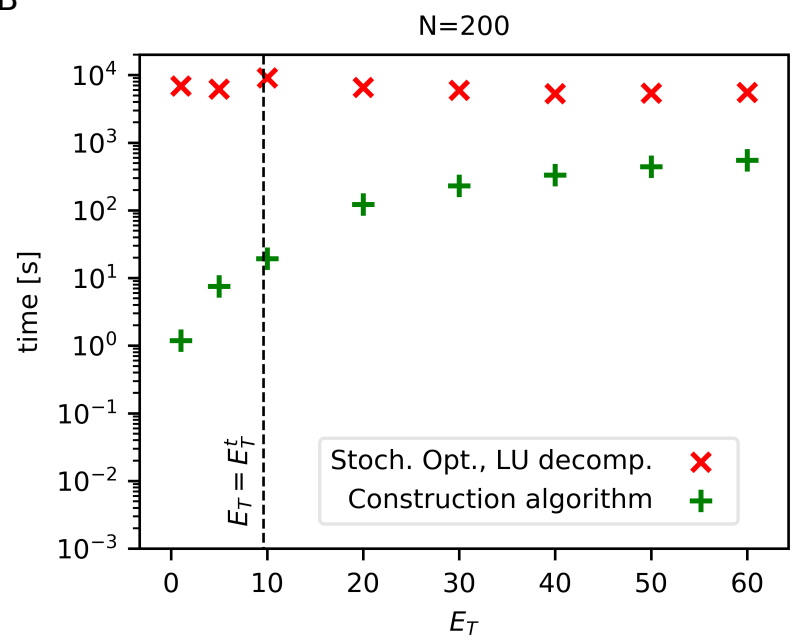

Figure G.2. Computational time vs the total number of enzymes in the system for different algorithms. We mark the computational time spent by the construction algorithm derived in this work (green pluses) and the average time spent by a stochastic optimization algorithm using either a tridiagonal symmetric solver (blue circles) or an LU-decomposition algorithm (red crosses). The system under consideration is a 1D system with two sources of substrate, one for each boundary. In (A) and (B) we consider a number of lattice sites of $N=100$ and $N=200$ respectively. The construction algorithm out-competes the stochastic optimization algorithm with LU-decomposition, which is the standard solver used in systems with no specific symmetries and higher dimensions. The construction algorithm can be a factor of 10 slower than the stochastic optimization algorithm with a tridiagonal solver. Such a solver however can be used only for specific systems, e.g. a 1D system with a tridiagonal diffusion operator. The optimizations were conducted on a single core of a Intel(R) Xeon(R) CPU E5-2640 v3. The average time spent by the stochastic optimization is taken over 20 repeats, the standard deviation is not shown as it is smaller than the size of the markers used.

${ }_{934}$ the more general setups, one would typically use an LU-decomposition to solve Eq. G9 for 935 linear reactions in the stochastic optimization algorithm. Such solver is $o\left(N^{3}\right)$, implying that 936 the stochastic optimization algorithm becomes $o\left(N^{5}\right)$. In Fig. G.2A,B the computational 937 time of the stochastic optimization algorithm using a LU-decomposition is plotted with red ${ }_{938}$ crosses for $N=100$ and $N=200$ respectively. We can see how the construction algorithm 939 out-competes the stochastic optimization one for all $E_{T}$ values and that the higher is the 940 amount of lattice sites $N$, the larger is the difference in computational times as expected 
A

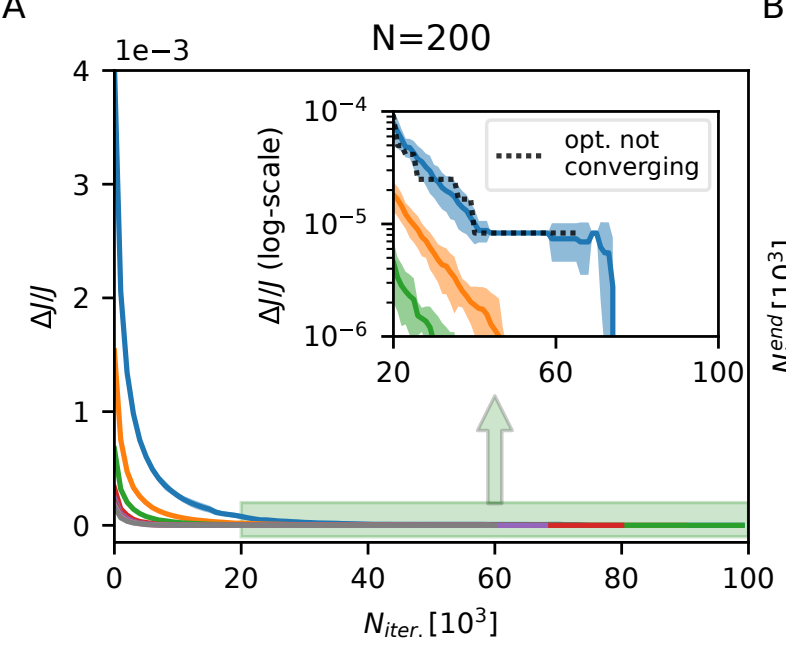

B

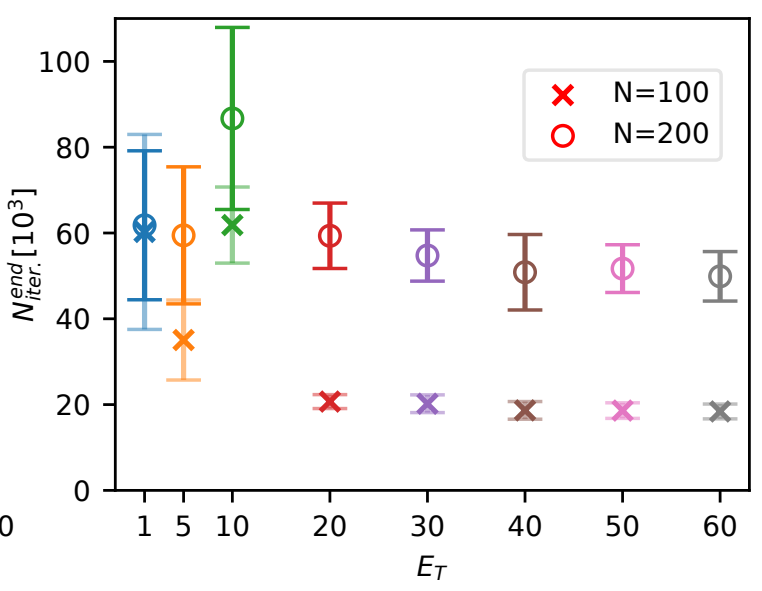

Figure G.3. Flux comparison and convergence of the stochastic optimization algorithm vs. the construction algorithm. (A) We plot the relative difference of fluxes $\Delta J / J=1-J_{\text {s.o. }} / J_{\text {c.a. }}$ as a function of the number of iterations taken in the stochastic optimization algorithm for different $E_{T}$ values and a discretization of $N=200$. Different colors correspond to different $E_{T}$ values as given by the points in panel (B). Some of the stochastic optimizations do not converge to $J_{c . a}$. For example, 16 out of 20 optimization runs get stuck at $\Delta J / J \approx 10^{-5}$ for $E_{T}=1$. We plot an example of such trajectory with a black dashed line in the inset of panel (A). The optimization stops before reaching our imposed limit of $N_{\text {iter. }}=10^{5}$ because the flux does not increase for $10^{4}$ iterations. The shaded areas correspond to the standard deviation of $\Delta J / J$ over 20 repeats. (B) We plot the average and the standard deviation of the number of iterations needed for the stochastic optimization to end $N_{i t e r}^{\text {end }}$. The stochastic optimization for $N=200$ gets about 3-times higher than the number of iterations needed for $N=100$ for $E_{T} \geq 20$. The $N_{i t e r}^{\text {end }}$ for the two discretizations get similar as $E_{T}$ decreases. This is due to the non-convergence of the stochastic optimization at low $E_{T}$ values for $N=200$. The stochastic optimization gets stuck at sub-optimal enzyme configurations and it is stopped after $10^{4}$ iterations with no flux increase.

${ }_{941}$ from the scaling argument. We therefore expects the construction algorithm to always be 942 convenient as compared to the stochastic optimization algorithm unless the systems present 943 simple geometries with specific symmetries. Then the construction algorithm could be slower 944 by a factor of 10 for large $E_{T}$ values.

945 The construction optimization algorithm has also the advantage of being exact and certain 946 to converge to the optimal solution. This is not always the case for the stochastic optimiza- 
947 tion algorithm, which may or may not converge to the global optimum. In Fig. G.3A, we 948 plot the relative difference between the two fluxes $\Delta J / J=1-J_{\text {s.o. }} / J_{\text {c.a. }}$, where $J_{\text {s.o. }}$ is the 949 flux obtained via the stochastic optimization and $J_{c . a}$. the one of the construction algorithm. ${ }_{950}$ We plot $\Delta J / J$ for different $E_{T}$ values as a function of the number of iterations taken in the 951 stochastic optimizations. None of the realizations, both for $N=100$ and $N=200$ resulted 952 in negative values of $\Delta J / J$, i.e. $J_{\text {s.o. }}>J_{\text {c.a. }}$. In the case of $N=100$, for any $E_{T}$ value and 953 for all the repeats, $\Delta J / J$ goes to zero. However, in the case of $N=200$ (Fig. G.3A), the 954 stochastic optimization does not always converge to the flux obtained via the construction 955 algorithm $J_{\text {c.a. }}$. For example, in the case of $E_{T}=1,16$ out of 20 repeats do not converge 956 to $J_{\text {c.a. }}$ and they get stuck at a value of $\Delta J / J \approx 10^{-5}$. We plot an example of such an 957 optimization trajectory in the inset of Fig. G.3A (black dotted line) in log-scale. For such a 958 trajectory, the stochastic optimization algorithm stops after $N_{i t e r} \approx 6 \cdot 10^{4}$. This is better 959 shown in Fig. G.3B, where we plot the number of iterations needed to end the stochastic 960 optimization, $N_{i t e r}^{\text {end }}$, for different $E_{T}$ values. We can see that for a discretization of $N=200$, ${ }_{961} N_{\text {iter. }}^{\text {end }}$ is about 3 -times higher as compared to the iterations needed for $N=100$ for $E_{T} \geq 20$. ${ }_{962}$ As $E_{T}$ decreases, the values of $N_{i t e r}^{\text {end }}$ of the two discretizations get closer to each other. This 963 is because in this regime the stochastic optimization for $N=200$ do not always converge to 964 the optimal enzyme arrangement. It gets stuck at some sub-optimal configuration, causing 965 the optimization to end prematurely after $10^{4}$ iterations with no flux increase.

\section{Appendix H: Betting games with coupled outcomes and bets}

\section{7 \\ 1. Gambler repeatedly betting a fixed amount of resources}

In the main text, we show that the optimal enzyme spatial allocation problem can be 969 mapped to a betting game where a gambler bets a fixed amount of resources at every 970 repetition of a game. In case of victory, the gambler wins an amount proportional to the 971 bet $b_{i}$ on the winning event $i$, times the odds payed back to the winner $\alpha_{i}$, i.e. an amount 972 of money $\alpha_{i} b_{i}$. The long-term amount of capital won by the gambler grows arithmetically 973 and is $C=\sum_{i} \alpha_{i} b_{i} p_{i}$, where $p_{i}$ is the probability that $i$ is the winning outcome. Betting a 974 fixed amount of resources at every repetition of the game implies that $\sum_{i} b_{i}=B$. We can 975 therefore ask: what is the optimal way to repartition the bets $b_{i}$ under the budget constraint? 
976 To answer the question we can look at the maximum of the following Lagrangian

$$
\mathcal{C}=\sum_{i} \alpha_{i} b_{i} p_{i}-\lambda\left(\sum_{i} b_{i}-B\right)
$$

977 The Lagrangian is linear with respect to the bets $b_{i}$. This implies that the optimal solution 978 is to bet all the budget on the outcome that has the highest expected odds $\alpha_{i} p_{i}$. Therefore 979 in this simple scenario the gambler never diversifies the bets placed. By looking at the 980 derivatives of the constrained capital with respect to the bets, we can see how they do not 981 depend on any of the bets $b_{i}$ and are given by the sum of two terms:

$$
\frac{\partial \mathcal{C}}{\partial b_{i}}=\alpha_{i} p_{i}-\lambda
$$

${ }_{982}$ The derivative above can be interpreted as the variation of the constrained capital per extra 983 bet placed. The first term represents the net expected gain $\alpha_{i} p_{i}$ per extra bet placed on 984 the outcome $i$, if no constraint was considered. It is equivalent to the first term present in 985 the derivative $\frac{\delta \mathcal{L}}{\delta e(\mathbf{r})}$, Eq. 7 of the main text, corresponding to the increase in reaction flux 986 that would be observed upon adding extra enzymes at position $\mathbf{r}$, if the constraints on the ${ }_{987}$ reaction-diffusion dynamics and the enzyme budget are neglected. The second term $-\lambda$ is 988 the marginal cost of adding extra capital and it is analogous to the $-\lambda_{e}$ term of $\frac{\delta \mathcal{L}}{\delta e(\mathbf{r})}$.

989 What we are missing is an equivalent of the second term of $\frac{\delta \mathcal{L}}{\delta e(\mathbf{r})},-\lambda_{V}(\mathbf{r}) k_{\text {cat }} F[\rho(\mathbf{r})]$, which 990 is generated by the coupling between $e(\mathbf{r})$ and $\rho(\mathbf{r})$ through the reaction-diffusion equation. 991 In fact, in the enzyme spatial allocation problem there is a dependency between the enzymes 992 allocated and the reaction flux generated, i.e. a dependency between the resources invested 993 in the system and the returns obtained. We can therefore ask: what would happen to our 994 betting problem if a feedback between the bets placed $b_{i}$ and the expected odds $\alpha_{i} p_{i}$ is 995 introduced?

996 In our mapping, the bets placed on a certain outcome correspond to the enzymes allocated 997 at a certain position, $b_{i} \leftrightarrow e(\mathbf{r})$, while the fixed budget corresponds to the total enzyme 998 amount, $B \leftrightarrow E_{T}$. The probability of $i$ being the winning event maps to the probability of 999 an enzyme being bound with the substrate at a certain position, $p_{i} \leftrightarrow F[\rho(\mathbf{r})]$, and the odds 1000 correspond to the catalytic rate, $\alpha_{i} \leftrightarrow k_{\text {cat }}$. Since in our correspondence $\alpha_{i}$ is constant, we 1001 introduce a feedback between the bets $b_{i}$ and the expected odds $\alpha_{i} p_{i}$ by considering each $p_{i}$ 1002 to be a function of the vector of bets $p_{i}=f_{i}(\mathbf{b})$. Note, however, that a feedback could also 1003 be constructed by having the odds $\alpha_{i}$ function of the bets $\mathbf{b}$. A new functional $\mathcal{C}_{f b}$ can be 
1004 considered:

$$
\mathcal{C}_{f b}=\sum_{i} \alpha_{i} b_{i} p_{i}-\sum_{i} \lambda_{i}\left[f_{i}(\mathbf{b})-p_{i}\right]-\lambda\left(\sum_{i} b_{i}-B\right),
$$

1005 where $\lambda_{i}$ are the Lagrange multipliers corresponding to the constraints on the probabilities $1006 p_{i}$ being functions $f_{i}(\cdot)$ of the bets placed $\mathbf{b}$. The role played by the $\lambda_{i}$ is analogous to the 1007 one played by $\lambda(\mathbf{r})$ in the Lagrangian $\mathcal{L}$ Eq. 5 of the main text. When we take derivatives 1008 of $\mathcal{C}_{f b}$ with respect to $b_{i}$ we now have extra terms:

$$
\frac{\partial \mathcal{C}_{f b}}{\partial b_{i}}=\alpha_{i} p_{i}-\sum_{j}\left(\lambda_{j} \frac{\partial f_{j}(\mathbf{b})}{\partial b_{i}}\right)-\lambda
$$

1009 The contribution of the terms in the sum $\sum_{j}(\cdot)$ comes from the coupling between the bets 1010 placed and the returns generated, analogously to the second term present in the derivative $1011 \frac{\delta \mathcal{L}}{\delta e(\mathbf{r})}$, Eq. 7 of the main text. The exact form of $f_{i}(\mathbf{b})$ depends on the details of the model 1012 considered for the game played by the gambler.

${ }_{1013}$ For an optimal allocation of capital the derivative $\frac{\partial \mathcal{C}_{f b}}{\partial b_{i}}=0$, meaning that the sum of the 1014 first term with the sum $\sum_{j}(\cdot)$ equals the marginal cost $\lambda$, in a similar way as what we found 1015 for the optimal allocation of enzymes by imposing $\frac{\delta \mathcal{L}}{\delta e(\mathbf{r})}=0$. It results optimal to invest 1016 up to the point for which the net marginal gain expected from our investment (including 1017 the contribution due to the feedback) equals the marginal cost of adding extra resources. 1018 Moreover, diversification of bets can be optimal depending on the functional form of $f_{i}(\cdot)$. 1019 For example, let's consider the case of two possible winning outcomes plus a third outcome 1020 having nonzero probability of happening but zero odds. In this simple scenario and in the 1021 case of bets affecting only the correspondent outcomes, i.e. $\frac{\partial f_{i}}{\partial b_{j}}=0$ with $i \neq j$, the optimal 1022 strategy is

$$
b_{1}^{*}=-\frac{\alpha_{1} f_{1}-\alpha_{2} f_{2}-\alpha_{2} B \frac{\partial f_{2}}{\partial b_{2}}}{\alpha_{1} \frac{\partial f_{1}}{\partial b_{1}}+\alpha_{2} \frac{\partial f_{2}}{\partial b_{2}}}, \quad b_{2}^{*}=B-b_{1}^{*}, \quad b_{3}^{*}=0 .
$$

${ }_{1023}$ The optimal strategy results in diversified bets (i.e. $b_{1}^{*}>0, b_{2}^{*}>0$ ), if, e.g., the events 1024 have diminishing returns $\left(\frac{\partial f_{i}}{\partial b_{i}}<0\right.$, with $\left.i=1,2\right)$ and at the same time an event has higher 1025 expected odds as compared to the other $\left(\alpha_{1} f_{1}>\alpha_{2} f_{2}+\alpha_{2} B \frac{\partial f_{2}}{\partial b_{2}}\right)$. The term $\alpha_{2} B \frac{\partial f_{2}}{\partial b_{2}}$ accounts 1026 for how the expected odds of the second event change due to the diminishing returns effect. ${ }_{1027}$ Alternatively one could have diversified bets if the expected odds of an event are lower than 1028 the other $\left(\alpha_{1} f_{1}<\alpha_{2} f_{2}+\alpha_{2} B \frac{\partial f_{2}}{\partial b_{2}}\right)$ and the events present an increasing returns effect for 1029 which the probabilities of the events of being the winning ones increase with the amount of 1030 bets placed on them $\left(\frac{\partial f_{i}}{\partial b_{i}}>0\right.$, with $\left.i=1,2\right)$. Moreover, if the events behave differently and 
1031 for example an event presents diminishing returns and the other increasing returns, we still 1032 get optimal diversification of bets as given by Eq. H5.

1033 To see how such optimal diversification strategies vary as a function of the total budget ${ }_{1034} B$, one can use the construction algorithm presented in the main text and derived in Ap${ }_{1035}$ pendix G. The algorithm consists in looking at the Hessian matrix of the capital $\mathcal{C}_{f b}$ with 1036 respect to the bets placed on different outcomes, as similarly illustrated in the main text for 1037 the enzyme spatial allocation problem. The algorithm applies to any game having a linear 1038 budget constraint. This means that it can be applied to investment problems where the 1039 revenue grows arithmetically as in Eq. H3, but also problems where the revenue grows expo1040 nentially as it is the case for example for the famous Kelly problem [10]. There, instead of 1041 considering the Hessian matrix of the capital as given by $\mathcal{C}_{f b}$, one would look at the Hessian 1042 of the capital growth rate with respect to the bets placed at different outcomes and would 1043 optimize for the growth rate as the budget gradually increases.

\section{2. Gambler repeatedly reinvesting a fraction of the resources - Generalization of 1045 Kelly's criterion}

${ }_{1046}$ In this section we generalize Kelly's optimal betting criterion [10] for a gambler that 1047 wants to maximize his steady state capital growth rate $G$ by betting on a series of mutually 1048 exclusive events. In the original formulation of Kelly, the capital growth rate $G$ has the 1049 following form:

$$
G=\sum_{i} p_{i} \log \left[k+b_{i} \alpha_{i}\right]
$$

1050 where $p_{i}$ is the probability for the event $i$ to be the winning one, $k$ is the fraction of the 1051 gambler's capital kept, $b_{i}$ is the fraction of gambler's capital invested on the event $i$ and $\alpha_{i}$ 1052 are the odds, i.e. the money payed back to the gambler in case $i$ is the winning event.

1053 Kelly's aim was to determine the optimal way of partitioning the gambler's capital given 1054 that the gambler cannot borrow nor lend money, hence his aim was to determine the optimal 1055 set of $b_{i}$ and $k$, under the constraint $\sum_{i} b_{i}+k=1$. We can therefore consider the functional

$$
\mathcal{G}=\sum_{i} p_{i} \log \left[k+b_{i} \alpha_{i}\right]-\lambda\left[\sum_{i} b_{i}+k-1\right]
$$

1056 where $\lambda$ is the Lagrange multiplier associated with the constraint on the gambler's capital. 
To find the optimal set of $b_{i}$ and $k$ we perform the following derivatives:

$$
\begin{gathered}
\frac{\partial \mathcal{G}}{\partial b_{i}}=\frac{p_{i} \alpha_{i}}{k+\alpha_{i} b_{i}}-\lambda=0 \quad \text { for } i \in s \\
\frac{\partial \mathcal{G}}{\partial k}=\sum_{i}\left[\frac{p_{i}}{k+\alpha_{i} b_{i}}\right]-\lambda=0 \\
\frac{\partial \mathcal{G}}{\partial b_{i}}=\frac{p_{i} \alpha_{i}}{k}-\lambda \leq 0 \quad \text { for } i \in s^{\prime}
\end{gathered}
$$

where we indicated with $s$ the set of events for which it is optimal to bet and $b_{i}>0$, and with $s^{\prime}$ the set of events for which betting is suboptimal and $b_{i}=0$. The derivatives above can be interpreted as the variation of the constrained capital growth rate $\mathcal{G}$ per extra bet placed H8, H10 or per extra resource kept H9. The first terms of the derivatives are the net marginal increase of $G$ generated by either placing the bets H8, H10 or by keeping an extra amount of resources H9, if no constraint was considered. The $-\lambda$ terms correspond to the marginal cost of adding the extra resources, analogously to the $-\lambda_{e}$ term present in the derivative $\frac{\delta \mathcal{L}}{\delta e(\mathbf{r})}$, Eq. 7 of the main text. Analogously to the enzyme spatial allocation problem, we see that the optimal strategy is to bet on those events $i \in s$ up to the point for which the marginal gain generated equals the marginal cost. Moreover the optimal way of repartitioning the gambler's capital, known as Kelly criterion, can be obtained by solving for the above derivatives, together with the use of the constraint:

$$
\begin{gathered}
\lambda=1 \\
k=\frac{1-\sum_{i \in s} p_{i}}{1-\sum_{i \in s} 1 / \alpha_{i}} \\
b_{i}=\frac{p_{i} \alpha_{i}-k}{\alpha_{i}} \quad \text { for } i \in s .
\end{gathered}
$$

${ }_{1057}$ To understand how to determine the set $s$ of events for which it is optimal to bet, we refer 1058 the readers to Kelly's original paper [10], here we just say that finding the optimal $s$ is 1059 similar in determining the positions at which is optimal to allocate enzymes in the class of 1060 reaction-diffusion systems considered in the main text.

1061 Again, the variation of the constrained quantity we want to maximize per extra resource 1062 invested, i.e the derivative Eq. H8, is given by the sum of just two terms and it lacks an 1063 equivalent of the second term of $\frac{\delta \mathcal{L}}{\delta e(\mathbf{r})},-\lambda_{V}(\mathbf{r}) k_{\text {cat }} F[\rho(\mathbf{r})]$, which is generated by the coupling 1064 between $e(\mathbf{r})$ and $\rho(\mathbf{r})$ through the reaction-diffusion equation. We can imagine to add a 1065 dependency between the probabilities of winning and the bets placed, by assuming that 
1066 the probabilities $p_{i}$ are now functions of the bets placed $p_{i}=f_{i}(\mathbf{b})$, where $\mathbf{b}$ is the vector $1067\left(b_{1}, b_{2}, \ldots\right)$. A new functional $\mathcal{G}$ can be considered:

$$
\mathcal{G}=\sum_{i} p_{i} \log \left[k+b_{i} \alpha_{i}\right]-\lambda\left[\sum_{i} b_{i}+k-1\right]-\sum_{i} \lambda_{i}\left[p_{i}-f_{i}(\mathbf{b})\right]
$$

1068 where $\lambda_{i}$ are the Lagrange multipliers corresponding to the constraints on having the proba1069 bilities functions of the bets placed. The role played by the $\lambda_{i}$ is analogous to the one played 1070 by $\lambda(\mathbf{r})$ in the Lagrangian $\mathcal{L}$ Eq. 5 . When we take the derivatives of $\mathcal{G}$ with respect to $b_{i}$ we 1071 now have extra terms:

$$
\frac{\partial \mathcal{G}}{\partial b_{i}}=\frac{p_{i} \alpha_{i}}{k+\alpha_{i} b_{i}}+\sum_{j}\left(\lambda_{j} \frac{\partial f_{j}(\mathbf{b})}{\partial b_{i}}\right)-\lambda=0
$$

1072 The sum $\sum_{j}(\cdot)$ comes from the coupling between the bets placed and the returns generated, 1073 analogously to the second term present in the derivative $\frac{\delta \mathcal{L}}{\delta e(\mathbf{r})}$, Eq. 7 of the main text. The 1074 exact form of $f_{i}(\mathbf{b})$ depends on the details of the model considered for the game played by 1075 the gambler.

1076 For an optimal allocation of capital the derivative $\frac{\partial \mathcal{G}}{\partial b_{i}}=0$, meaning that the sum of the 1077 first term with the sum $\sum_{j}(\cdot)$ equal the marginal cost $\lambda$, in a similar way as what we found 1078 for the optimal allocation of enzymes by imposing $\frac{\delta \mathcal{L}}{\delta e(\mathbf{r})}=0$.

1079 Since the cost of adding extra resources is constant and equals $-\lambda$, we can use a similar 1080 construction algorithm as the one presented in the main text to find the optimal gambling 1081 strategy. Instead of considering the Hessian of the reaction flux with respect to the addition 1082 of enzymes at different positions, one needs to compute the Hessian of the capital growth 1083 rate with respect to the bets placed on different outcomes. Once the Hessian is found, one ${ }_{1084}$ can derive the optimal way of repartitioning the gambler's bet to track the optimal $G$ as the 1085 total budget gradually increases in exactly the same way as we did for the enzyme spatial 1086 allocation problem. 